\title{
The impact of system noise in polarimetric SAR imagery on oil spill observations
}

\author{
Martine M. Espeseth, Camilla Brekke, Member, IEEE, Cathleen E. Jones, Member, IEEE, Benjamin \\ Holt, Member, IEEE, and Anthony Freeman, Fellow, IEEE
}

\begin{abstract}
The effects of both system additive and multiplicative noise on X-, C-, and L-band Synthetic Aperture Radar (SAR) data covering oil slicks are examined. Prior studies have attempted to characterize such oil slicks, primarily through analysis of polarimetric SAR data. Here we factor in system noise that is added to the backscattered signal, introducing artifacts that can easily be confused with random and volume scattering. This confusion occurs when additive and/or multiplicative system noise dominates the measured backscattered signal. Polarimetric features used in this study are shown to be affected by both additive and multiplicative system noise, some more than others. This study highlights the importance of considering specifically multiplicative noise in the estimation of the signal-to-noise ratio (SNR). The SNR based on additive noise should at least be above 10dB. The SNR involving both additive and multiplicative noise should at least be above OdB. The SNR from TerraSAR-X and Radarsat-2 is below $0 \mathrm{~dB}$ for the majority of the oil slick pixels when considering both the additive and multiplicative noise, rendering these data unsuitable for any analysis of the scattering properties and characterization. These results are in contrast to the reduced impact of noise on oil slicks detected by the Lband UAVSAR system. In particular, we find there is no need to invoke exotic scattering mechanisms to explain characteristics of the data. We also recommend a noise subtraction for any polarimetric scattering analysis.
\end{abstract}

Index Terms-Synthetic Aperture Radar, UAVSAR, Radarsat2, TerraSAR-X, SNR, additive noise, multiplicative noise, oil spill

\section{INTRODUCTION}

Polarimetric Synthetic Aperture Radar (SAR) data has been utilized in multiple remote sensing investigations of marine oil spills (see, e.g., [1]-[3]). Most studies have focused on oil slick detection, in an effort to identify polarimetric features that demonstrate high detection capabilities for different kinds of oil under various wind and ocean conditions (see, e.g., [1], [4][6]). After a slick has been detected, investigators then seek to extract more information about the oil slick. Such characterization of oil slicks includes extraction of physical properties such as the dielectric constant, the volumetric fraction of the oil in an oil-water mixture, and distinguishing between various types of oil. Studies by [2], [3], [7] have addressed the potential of polarization diversity in a SAR system to

M. M. Espeseth and C. Brekke are with the Department of Physics and Technology, UiT The Arctic University of Norway, 9037 Troms $\varnothing$, Norway.

C. E. Jones, B. Holt, and A. Freeman are with the Jet Propulsion Laboratory, California Institute of Technology, Pasadena, CA 91125 USA

Manuscript received Month XX, 201X; revised Month XX, 201X; accepted Month X, 201X. Date of publication Month XX, 201X; date of current version Month X, 201X. (Corresponding author: M. M. Espeseth (martine.espeseth@uit.no)) yield this kind of information about the characteristics of the oil, which could be very important in a potential oil spill recovery, as knowledge about, e.g., the oil type, or the internal variations within the oil slick might facilitate more efficient clean-up operations. Oil slick characterization is only possible if signal backscattered from the oil slick is separable within the measured signal. Unfortunately, oil slicks seen in polarimetric SAR data are particularly susceptible to misinterpretation due to noise contamination of their low backscatter values, and the importance of properly evaluating the noise is emphasized.

The measured signal in all SAR systems contains noise in addition to the signal of interest, which is the normalized radar-cross-section (RCS), $\left(\sigma^{0}\right)$, of the target. In general, there are three imaging effects to consider when attempting to recover the RCS. These are listed in [8] and are as follows; (i) scaling of the RCS due to propagation, antenna pattern, and processing effects; (ii) spatial correlation induced by the processing; and (iii) bias in the estimated RCS due to system noise. Examples of potential noise sources that can contribute to the additive noise power (the bias in the estimated RCS) are the thermal noise and quantization noise from the analogto-digital conversion (ADC) [9].

Freeman [9] demonstrated the effect of additive noise on polarization signatures for a typical Bragg scatterer, and discussed the effect of this type of noise on the radar measurements. Several studies have conducted a "noise analysis", containing information about the signal-to-noise ratio (SNR) of the various polarization channels of different investigated mediums like an oil slick, clean sea, biogenic slick, etc. (see, e.g., [2], [10], [11]). In these studies, the authors usually verify whether the measured signal is above the noise floor, and further evaluate the need to discard some of the polarization channels (often the cross-polarization channel is discarded for oil slick analysis). The noise floor is often set to the noiseequivalent-sigma-zero (NESZ) that is given in the product file of the data. However, even though the signal is above the noise floor, the measured signal still contains noise. Minchew et al. [10] identified that the measured signal should be $6 \mathrm{~dB}$ above the noise floor, while [12] identified this limit to be between $7-8 \mathrm{~dB}$. Both studies concluded this level above the noise floor based on the measured intensity values as a function of the incidence angle. For oil slick characterization and extraction of physical properties using polarimetric SAR data, these results imply that the backscattered signal needs to be well above the noise power, i.e., noise floor.

It is possible to detect oil slicks so long as the oil/sea backscatter contrast is high, which means the backscatter from 
the oil slick can be close to the noise floor of the radar, provided the ocean surface return is well above the noise floor. Unfortunately, instrumental noise might still influence the detection capability using SAR, which was demonstrated by Angelliaume et al. [13] using an oil slick detection algorithm. In the same study, the authors added noise to a scattering vector from a high SNR airborne instrument with full-polarimetric capability and calculated the probability of detection using a set of polarimetric features. They discovered that the performance of the oil slick detection for some polarimetric features was severely deteriorated by the instrumental additive noise.

When studying oil slicks with SAR, multiplicative noise has traditionally not been considered (see, e.g., [5], [10], [13][16]). The multiplicative noise raises the noise floor, hence reducing the overall SNR in the data. The main objective of this study is to understand the sensitivity to both additive and multiplicative noise power for a set of well known and commonly used polarimetric features for observing marine oil slicks. More specifically, the analysis covers (1) how the feature values behave as a function of both the simulated noise and true noise (additive and multiplicative) within the radar measurements; (2) identifying the minimum SNR below which the data are too contaminated by the noise to provide reliable information about the surface properties from oilcovered surfaces; and (3) exploring the feature behavior when the additive noise power is subtracted from the second order sample covariance and coherency matrix. The analysis is based on quad-polarimetric data from the L-band sensor Uninhabited Aerial Vehicle Synthetic Aperture Radar (UAVSAR), the C-band sensor Radarsat-2 (RS-2), and dual-polarimetric (two copolarization channels) data from the X-band sensor TerraSAR-X (TS-X) obtained off Norway during a series of oil-on-water field campaigns.

\section{NOISE ARTIFACTS IN SAR}

As highlighted in Section I, various imaging effects have to be taken into consideration when recovering the RCS. The upcoming discussion includes the various artifacts and noise introduced in SAR, with a special focus on marine oil spills. This section also discusses the measures that are applied in this study for considering some of the noise/artifacts impacting the measured backscattered signal.

\section{A. Aliasing}

Well-known artifacts that can cause confusion in interpreting a SAR image are ambiguities due to aliasing in both the azimuth and range direction [9]. Aliasing in the range direction is caused by simultaneously receiving different pulses [9]. Azimuth ambiguities are caused by discrete (pulsed) sampling of the along-track or azimuth Doppler variations in the radar data and result from sidelobes that extend beyond the width of the main lobe of the SAR antenna in that direction, and are therefore at higher Doppler values [17]. The degree of aliasing and the separation distance at which they occur is controlled by the pulse repetition frequency (PRF) [17]. As a result of azimuth aliasing the signal from any target is repeated (maybe several times) with a fraction of the main signal equal to the azimuth ambiguity ratio. For bright targets such as ships these ambiguities are sometimes observed as ghosts in the SAR image. In this study we have selected oil spill areas that are not contaminated by azimuth ambiguities from ships. Nevertheless, these darker areas may be corrupted by ambiguous signals from nearby clean sea areas, that are brighter than the oil spill. The contrast between oil and sea can be high, and therefore a fraction of the SAR signal from the surrounding clean sea will be present in oil-covered pixels.

Table I shows the ambiguity levels for the three sensors investigated. The total ambiguity ratio in TS-X is $<-16 \mathrm{~dB}$ [18] for the stripmap dual-polarimetric mode (used in this study). This ratio was in [18] calculated based on the average ratio between the signal power and the aliased power that is caused by azimuth and range ambiguities for one pixel. For RS2 the azimuth and range ambiguities are usually both -35 $\mathrm{dB}$ [19], except for the higher incidence angles, for which the range ambiguity drops to $-25 \mathrm{~dB}$. Range ambiguities are usually not significant noise sources for airborne systems such as UAVSAR, and typical azimuth ambiguity levels are estimated at $-24 \mathrm{~dB}$ [Scott Hensley, personal communication, May 2019]. In this study the ambiguity levels are factored into the multiplicative noise ratio when estimating the SNR.

\section{B. Cross-talk}

For polarization diverse data, cross-talk and channel imbalance between the various polarization channels are unavoidable because of leakage between the polarization channels. An extra processing step (see, e.g., [20], [21]) involving for example assumptions about the scattering medium, is sometimes performed to reduce cross-talk by balancing the amplitude and the phase between channels. The most significant, and most readily observed, effect of unbalanced polarization channels, due to cross-talk, will be higher apparent levels of $\mathrm{HV}$ backscatter. In cases of severe cross-talk the target signal for each polarization channel may be contaminated and alter the interpretation and estimation of physical parameters using a given model (e.g., tilted Bragg model [22]).

In Table I, the estimated cross-talk value for RS-2 is $32 \mathrm{~dB}$ ([23], [24]), but various values between $-30 \mathrm{~dB}$ and $40 \mathrm{~dB}$ have been reported (see, e.g., [23], [25]). The estimated cross-talk prior to any cross-talk calibration of the UAVSAR is generally on the order of $-30 \mathrm{~dB}$ [26]. The antenna cross-talk can only be corrected if the quad-polarimetric scattering matrix is available (see, e.g., [27], [28]). Cross-talk removal has been performed on the quad-polarimetric Radarsat-2 products prior to receiving the data [23]. The HH-VV dual-polarimetric mode of TerraSAR-X is used for this study, where the two cross-polarization channels are not acquired. Hence, cross-talk between the two copolarization channels are insignificant. No cross-talk removal is performed on the UAVSAR data.

\section{Effects from sidelobes}

While measuring the response from the surface, sidelobes might spatially smear the signal around a target. The peakto-sidelobe ratio (PSLR) and integrated sidelobe ratio (ISLR) 
TABLE I

AMBIGUITY LEVELS, CROSS-TALK, PSLR, ISLR, AND NESZ (ADDITIVE NOISE) OF THE UAVSAR, RS-2, AND TS-X. THE VALUES OF THE TS-X ARE FROM DUAL-POLARIZATION STRIPMAP.

\begin{tabular}{l|l|l|l} 
& UAVSAR & RS-2 & TS-X \\
\hline Ambiguity level [dB] & $\approx-24$ (az.) & \\
& & $-35 /-35$ (az./rg.) (FQ1-26) [19] & $<-16[18]$ \\
& $-35 /-25$ (az./rg.) (FQ28-31) & \\
Cross-talk [dB] & $-30[26]$ & $<-32[23],[24]$ & NA \\
PSLR [dB] & $-21^{b}[26]$ & $<-18[29]$ & $-25[18]$ \\
ISLR [dB] & $-17.67^{c}[26],[30]$ & $<-14.9[29]$ & $-18[18]$ \\
NESZ [dB] & -30 to $-50[26]$ & -31 to $-39[31]$ & $-19[18]$ \\
${ }^{a}$ Ambiguity level (azimuth (az.)) of UAVSAR was provided by Scott Hensley (JPL), personal communication 2019. \\
${ }^{b}$ The UAVSAR PSLR value was provided by Brian Hawkins (JPL) (personal communication 2019) using a weighting factor $\eta=0.5[26]$ \\
${ }^{c}$ The UAVSAR ISLR was calculated using equation (22) in [30] with a weighting factor $\eta=0.5[26]$.
\end{tabular}

measure the SAR performance in resolving a weak target (for example oil) in the presence of a strong target (for example ships/rigs). The PSLR is defined as the ratio between the height of the largest sidelobe and the height of the main lobe (expressed in $\mathrm{dB}$ ) [17]. The ISLR is the ratio of the integrated energy in the sidelobes to the amount of energy in the main lobe (also expressed in $\mathrm{dB}$ ) [17]. A high value (i.e., low ratio) in both the PSLR and ISLR indicate that the signal is smeared out along and across track, and could potentially generate a bright cross at a given point target (for example ships). Hence, low values (i.e., high ratio) of PSLR and ISLR are desirable, and the PSLR level is recommended to be approximately -20dB [17]. In Table I, TS-X and UAVSAR have acceptable values of the PSLR, namely $-25 \mathrm{~dB}$ and $21 \mathrm{~dB}$, respectively. The PSLR for RS-2 is $<-18 \mathrm{~dB}$ [29], which satisfies the tolerance level $(<-17 \mathrm{~dB})$ for oil spill detection identified in [32], but not the criteria set by [17]. There are some ships surrounding the oil slicks in some of the SAR scenes used in this study. Hence, the measured signal within the oil slick might be impacted by the sidelobes due to the response from the neighboring ships. In this study, this is mitigated by masking out the ships and its corresponding bright cross along several pixels in the range and azimuth direction prior to selecting the regions of interest (ROIs). Therefore, the oil slick regions studied here will not be located near the ships nor the bright cross. But the clean sea pixels surrounding the oil slick will still have a significant effect on the measured signal from the oil slick if the ISLR is high. This is because signals from nearby clean sea pixels could spillover (due to the sidelobes) into darker, oil-covered areas by a factor that adds up to be equivalent to the ISLR. The SNR for oil-covered pixels will then be reduced due to contributions from neighboring clean sea signals, which are not oil. The ISLR is therefore factored into the multiplicative noise ratio in estimation of the SNR for this study.

\section{Block adaptive quantization}

Another noise source is introduced by block adaptive quantization (BAQ) compression. The BAQ compression is performed on all three sensors evaluated in this study (UAVSAR [33], RS-2 [31], TS-X [18], [34]). The SAR signals are digitized using an ADC followed by the BAQ to reduce on-board data storage requirements and downlink rate [31]. The BAQ is a lossy data compression technique that introduces additive quantization noise in the data and depends on the backscattered signals in the scene. The key term here is "block": BAQ algorithms take a block of raw data and normalize to the average value of the quantized radar signals. The data are then re-quantized around that mean signal value. Thus an 8:3 BAQ algorithm behaves like a perfect 3-bit ADC, optimized so that "local" mean signal level for that block of samples falls right in the "sweet spot" of the ADC performance curve. The quad-polarization mode of RS-2 uses a 3-bit BAQ, which introduces a BAQ noise degradation that is $-14 \mathrm{~dB}$ lower than the mean signal level [31]. The quad-polarization modes of UAVSAR use an 8-bit BAQ [35]. BAQ quantization noise will reduce the SNR (see, e.g., [34], [36]), as a result of the adaptive scaling and re-quantization of the SAR signal [34]. The quantization noise contribution is relative to the mean signal level for the block of signals used [32]; for signal blocks where clean sea dominates the calculation of mean signal, the BAQ noise should be calculated relative to that level. The BAQ noise is already included in the additive noise power provided in TS-X [18]. The quantization noise is therefore considered for the UAVSAR and RS-2 when estimating the SNR.

\section{E. Thermal system noise}

The signal measured at the antenna must be larger than the thermal noise radiated in the radar system to enable an evaluation of the backscattering properties of the targets. The thermal noise is additive, and is usually considered the dominating factor in the NESZ and when calculating the overall scene SNR, where higher thermal noise results in reduced SNR. The thermal noise is assumed Gaussian white noise and is added to the observed signal. NESZ is defined in terms of the radar backscatter coefficient of an area that will appear at the same strength in the image as the thermal noise. For this study, the additive noise using the NESZ is considered for all sensors investigated when estimating the SNR, when simulating noise to the UAVSAR, and when subtracting the noise from the data with the objective of reducing the impact of the additive noise present in the polarimetric measurements. Typical NESZ (thermal noise) values for each sensor investigated in this study are shown in Table I. 


\section{AdDITIVE AND MULTIPLICATIVE NOISE IN POLARIMETRIC SAR}

For nominally calibrated polarimetric SAR data, the measured scattering matrix, $M$, is related to the target scattering matrix, $\boldsymbol{S}$, via [37]:

$$
M=S+N
$$

where $\boldsymbol{S}=\left[S_{H H}, S_{V H}, S_{H V}, S_{V V}\right]^{T}$ are the target scattering coefficients without noise (presumably), and $\boldsymbol{N}=$ $\left[n_{H H}, n_{V H}, n_{H V}, n_{V V}\right]^{T}$ represents the complex additive noise. The $\mathrm{H}$ represents horizontal and $\mathrm{V}$ is vertical polarization on either transmit or receive. The $\boldsymbol{N}$ is assumed to be complex Gaussian white noise with zero mean. The noise in the $\mathrm{HH}, \mathrm{VH}, \mathrm{HV}$, and VV polarization channel measurements are uncorrelated with each other and to the target scattering coefficients [9].

Equation (1) excludes the multiplicative noise factors, and to account for this, we suggest the following model for the measured RCS for clean sea and oil slicks;

$$
\sigma_{p q}^{0, m}=\sigma_{p q}^{0}+\sigma_{p q}^{n}+\sigma_{p q}^{A V G} M N R
$$

where $\sigma_{p q}^{0}$ is the RCS of the target, $p$ is the polarization on transmit, $q$ is the polarization on receive, and $\sigma_{p q}^{n}$ is the NESZ (additive noise power), i.e., $\left|n_{p q}\right|^{2}$. The $\sigma_{p q}^{A V G}$ is the average signal for each polarization channel that impacts the measured signal $\sigma_{p q}^{0, m}$. For ocean applications with small coverage and no land, the $\sigma_{p q}^{A V G}$ can be approximated to the mean signal level of homogeneous clean sea scatterers. The MNR is the multiplicative-noise-ratio (MNR) that factors in the ISLR, BAQ, and ambiguity-to-signal ratio (AMB). The $\sigma_{p q}^{0, m}=\left\langle\left|M_{p q}\right|^{2}\right\rangle$, where \langle\rangle denotes spatial averaging, and in this study a sliding $9 \times 9$ averaging window is used to reduce speckle, but it will not eliminate it. To be able to perform any useful characterization the $\sigma_{p q}^{0}$ from the oil slick must be larger than both the additive and multiplicative noise factors. This is determined using the SNR, and the upcoming section shows how the SNR is estimated using both the additive and the multiplicative noise components.

\section{A. Estimation of the SNR}

The SNR is an integral function of several sensor properties, including the gain on transmit and receive, carrier frequency, temperature, bandwidth, altitude and so forth. The $\mathrm{SNR}^{A}$ (signal-to-additive noise ratio) is estimated from the data and sensor properties and is based on the ratio between the RCS and the relative amount of the additive noise (NESZ), i.e.:

$$
S N R_{p q}^{A}=\frac{\sigma_{p q}^{0}}{\sigma_{p q}^{n}}=\frac{\sigma_{p q}^{0, m}-\sigma_{p q}^{n}}{\sigma_{p q}^{n}} .
$$

The SNR is equal to 1 (or SNR = 0dB) when the additive noise and the backscattered power are equal. The NESZ varies as a function of slant range (due to the antenna elevation pattern) and is the noise added to the observed signal. The NESZ is often given in the product file (nominal values) of the various sensors and the NESZ is normally at its lowest near mid-swath, resulting in a convex curve along the range profile.
The SNR in (3) excludes the multiplicative noise component (see (2)). Using the suggested model in (2), the SNR with both the additive and multiplicative noise, here named $\operatorname{SNR}^{A, M}$, can be expressed as

$$
S N R_{p q}^{A, M}=\frac{\sigma_{p q}^{0, m}-\left(\sigma_{p q}^{n}+\sigma_{p q}^{A V G} M N R\right)}{\sigma_{p q}^{n}+\sigma_{p q}^{A V G} M N R} .
$$

Here, $\sigma_{p q}^{A V G}$ is the average intensity in the scene, and MNR is defined as (in linear unit) [30];

$$
M N R=I S L R+1 / Q N R+A M B_{t}
$$

where $\mathrm{AMB}_{t}$ is the total ambiguity-to-signal ratio (range and azimuth). Since oil slicks are surrounded by clean sea, the signals from clean sea areas are repeated (due to aliasing) in the oil-covered areas by a factor equal to $\mathrm{AMB}_{t} \sigma_{p q}^{A V G}$ (aliased power). We assume here that azimuth ambiguities dominate the aliased signals. QNR is the quantization noise given as

$$
Q N R=10 \log _{10}\left(2^{2 N_{b}}\right)
$$

where $N_{b}$ is the number of bits. The QNR for RS-2 using the 3-bit BAQ is given as $-14 \mathrm{~dB}$ [31], whereas the QNR for the UAVSAR is estimated from the above equation. As mentioned in Section II, the QNR is already integrated in NESZ for TS$\mathrm{X}$, and is therefore not factored into the MNR. The nominal ISLR values of each sensor are shown in Table I, and these are the ones used. Note that the ISLR values for RS-2 are also given in the product file. Due to lack of information about how the ISLR is calculated, there are some uncertainties associated with these values. For example, whether the ratio given is for both range and azimuth or only one dimension. We treat the ISLR as the two-dimensional case (range and azimuth).

\section{SCATTERING PROPERTIES AND POLARIMETRIC SAR FEATURES}

This section discusses the relationship between the polarimetric SAR features and their interpretation with respect to the scattering properties.

Bragg scattering theory is often used to explain scattering from the ocean, where a relationship is established between the ocean surface roughness and its properties and the incoming electromagnetic wave [22], [38]. Various two-scale Bragg models have been explored that include both the small- and large-scale ocean surface roughness ([22], [39], [40]). The tilted Bragg model has been frequently used for modelling the backscatter from clean sea and oil (see, e.g., [3], [10]), and the $\mathrm{X}$-Bragg model has also been used for oil spill and sea surface studies (see, e.g., [1], [41]-[44]). The scattering process from a slick that dampens the capillary and small gravity ocean waves has often been called non-Bragg. The authors in [45] listed some possible scattering types that the literature considers non-Bragg. These are volumetric scattering, multiple scattering, double-bounce scattering, and a non-polarized component caused by the breaking waves and the surface film. The nonpolarized component was acknowledged as the most realistic explanation of the non-Bragg scattering [45]. Studies have demonstrated, using high SNR SAR data, that Bragg scattering also occurs within oil slicks (see, e.g., [10], [14]). 
Many different polarimetric features can be derived from polarimetric SAR data. In this study, we limit the choice of polarimetric features to those most frequently found in the literature, see Table II. The expected value of pure additive system noise, i.e., Gaussian white noise, is derived for each feature and is presented in Appendix A Table IV.

TABLE II

OverVIEW OF POlarimetric FEATURES INVESTIGATED. SEE E.G., [46] FOR THE CALCULATIONS OF THE PSEUDO PROBABILITIES $\left(p_{i}\right)$ AND EIGENVECTORS $\left(e_{i}\right)$ FROM THE COHERENCY MATRIX. SEE APPENDIX B FOR THE STOKES VECTOR $\left(S_{0}, S_{1}, S_{2}\right.$, AND $\left.S_{3}\right)$. $\phi_{H} H$ AND $\phi_{V V}$ ARE THE PHASES OF THE COMPLEX SCATTERING VECTORS $M_{H H}$ AND $M_{V V}$.

$$
\begin{array}{l|l}
P D=\sigma_{V V}^{0, m}-\sigma_{H H}^{0, m} & H=-\sum_{i=1}^{3} p_{i} \log _{3} p_{i} \\
r_{C O}=\Re\left(\left\langle M_{H H} M_{V V}^{\star}\right\rangle\right) & H_{C O}=-\sum_{i=1}^{2} p_{i} \log _{2} p_{i} \\
\gamma_{H H / V V}=\sigma_{H H}^{0, m} / \sigma_{V V}^{0, m} & \alpha=\sum_{i=1}^{3} p_{i} \cos ^{-1}\left(\left|\boldsymbol{e}_{i}(1)\right|\right) \\
\rho_{C O}=\frac{\left|\left\langle M_{H H} M_{V V}^{\star}\right\rangle\right|}{\sqrt{\left\langle\left|M_{H H}\right|^{2}\right\rangle\left\langle\left|M_{V V}\right|^{2}\right\rangle}} & D o P=\frac{\sqrt{S_{1}^{2}+S_{2}^{2}+S_{3}^{2}}}{S_{0}} \\
\sigma_{\phi_{C O}}=s t d\left(\phi_{H H}-\phi_{V V}\right) & \chi=\frac{1}{2} \sin ^{-1}\left(-\frac{S_{3}}{D o P S_{0}}\right)
\end{array}
$$

The polarization difference $(P D)$ has a low sensitivity to the incidence angle and look direction when observing an oil slick, as well as having a high oil-sea contrast [12]. Previous studies have observed low $P D$ values for low SNR areas like oil slick surfaces (see, e.g., [47]), and higher $P D$ for clean sea surfaces. According to [48], the backscattered intensities over the ocean are divided into two components; one polarized component associated with the two-scale Bragg model, and one non-polarized component. The non-polarized component is caused by wave breaking from steep and rough patches on the surface. The non-polarized component is removed in $P D$, and we are left with a difference between the Bragg scattering components of $\mathrm{HH}$ and VV [48].

The copolarization ratio $\left(\gamma_{H H / V V}\right)$ has been investigated in multiple studies (see, e.g., [2], [10], [47]), and according to the tilted Bragg scattering model, this feature is independent of the damping of small capillary waves by the oil. $\gamma_{H H / V V}$ is, in the tilted Bragg model, a function of the dielectric properties, the incidence angle, and the tilt angles [22]. The $\gamma_{H H / V V}$ is often used as input when estimating the dielectric constant to extract the volumetric fraction of oil in the oil-sea mixture. In order to extract the volumetric oil fraction, the system noise needs to be low in the two copolarization channels. The studies in [3], [49], [50] used low noise floor radars on airplanes to extract the oil fraction. Using spaceborne satellites with higher noise floor will present a challenge. For a high oil-sea contrast in this feature, the oil slick must be thick enough relative to the wavelength within the medium or have a high oil content in the oil-sea mixture. According to theoretical models, $\gamma_{H H / V V}$ is close to 1 at lower incidence angles, and decreases with increasing incidence angles [51]. Data-based estimates of $\gamma_{H H / V V}$ yield lower values for clean sea areas compared to an oil-covered surface [10]. Unfortunately, the presence of additive noise might result in higher $\gamma_{H H / V V}$ values for oil-covered areas due to low SNR. As the noise power increases and becomes much larger than the $\mathrm{HH}$ and VV intensities, $\gamma_{H H / V V}$ will tend towards 1 (assuming the noise in each polarization is at a similar level).
The real part of the copolarization cross product $\left(r_{C O}\right)$, the magnitude of the copolarization correlation coefficient $\left(\rho_{C O}\right)$, and the copolarized phase difference $\left(\phi_{C O}\right)$ are features that depend on $\left\langle M_{H H} M_{V V}^{\star}\right\rangle$. The $\left\langle M_{H H} M_{V V}^{\star}\right\rangle$ term is only independent of the noise if the noise power is decorrelated with the target scattering coefficients and the noise power from the other polarization channels (see, e.g., [9]), i.e.;

$$
\begin{aligned}
\left\langle M_{H H} M_{V V}^{\star}\right\rangle & =\left\langle\left(S_{H H}+N_{H H}\right)\left(S_{V V}^{\star}+N_{V V}^{\star}\right)\right\rangle \\
& =\left\langle S_{H H} S_{V V}^{\star}\right\rangle+\left\langle S_{H H} N_{V V}^{\star}\right\rangle \\
& +\left\langle N_{H H} S_{V V}^{\star}\right\rangle+\left\langle N_{H H} N_{V V}^{\star}\right\rangle \\
& =\left\langle S_{H H} S_{V V}^{\star}\right\rangle .
\end{aligned}
$$

Lower values of $r_{C O}$ and $\rho_{C O}$ have been observed for oil slicks compared to clean sea surfaces [2], consistent with what one would expect to see for pure random noise (see Table IV in Appendix A). Kasilingam et al. [52] reported that $\rho_{C O}$ is insensitive to changes in the short-scale roughness, and could therefore be sensitive to variation in the dielectric properties between clean sea and oil slicks. Low $\rho_{C O}$ values may imply depolarization effects. The expected value of $\rho_{C O}$ for pure noise is 0 . Depolarization in the backscattered signal may be due to the presence of complex surfaces, multiple-scattering surface layers, but may also be attributed to the presence of system noise [53]. The latter explanation is the one explored in this study.

The HH-VV phase difference $\left(\phi_{C O}\right)$ is not frequently used to examine oil spills with SAR, but its standard deviation is, i.e., $\sigma_{\phi_{C O}}$. The $\sigma_{\phi_{C O}}$ feature is another measure of the degree of correlation between $S_{H H}$ and $S_{V V}$. The expected value of $\phi_{C O}$ should be independent of the additive noise factor because the complex noise components measured in the various polarization channels decorrelate with each other and with the scattering coefficients (see (7)). However, $\sigma_{\phi_{C O}}$ will depend on the noise power. This is significant because the expected value of a given feature may be independent of the noise, but its variance might strongly depend on it. $\sigma_{\phi_{C O}}$ has been found to increase with the presence of oil (see, e.g., [54]-[57]), and this behavior was according to [54], [55] explained by the different scattering process (Bragg vs. nonBragg) between clean sea and oil. Some studies have showed that the broadening of $\phi_{C O}$ could be due to system noise (see, e.g., [10], [16], [43]). Minchew et al. [10] discovered no difference between the $\sigma_{\phi_{C O}}$ for oil and clean sea except in the presence of instrument noise for one UAVSAR scene. Alpers et al. [45] presented different theories on the broadening of the copolarization phase difference due to the presence of oil, for example; (1) the broadening might occur due to the fact that the Bragg waves are tilted by long waves; (2) instrument noise; (3) the inhomogeneity of the scattering medium due to irregular oil slick patches of various thickness. In [43], [58], it was suggested that the broadening of $\sigma_{\phi_{C O} O}$ was influenced by the system noise at high incidence angles $(\theta \approx 34)$ using TS-X images. In this study, we investigate whether this explanation is, in fact, the one most consistent with the observations.

Two types of entropy measures are evaluated, including a dual-polarized one that can be estimated from the TS$\mathrm{X}$ scenes. The first is the copolarization entropy $\left(H_{C O}\right)$ 
calculated from the sample $2 \times 2$ coherency matrix based on the $\mathrm{HH}$ and $\mathrm{VV}$ channels, and the second is the entropy $(H)$ calculated from the sample $3 \times 3$ coherency matrix (including the $H V$ channel). The entropy, $H \in\{0,1\}$, describes the randomness of the scattering, where $H=1$ indicates a random mixture of scattering mechanisms and $H=0$ represents a single scattering response [46]. Gaussian white noise gives an entropy close to 1 , and could therefore be confused with multiple and random scattering. Whether dominating or not, information about the scattering type is provided by the mean alpha angle $(\alpha) \in\left\{0,90^{\circ}\right\}$ : low $\alpha$ values represent surface scattering, intermediate $\alpha$ volume scattering, and high values double-bounce scattering [46], [59]. For pure random noise, $\alpha$ is $60^{\circ}$ [59], so if the additive noise term dominates, the mean $\alpha$ angle will be located in the intermediate $\alpha$ value range. Several oil spill studies have used the $H / \alpha$ method on SAR data from spaceborne satellites and found high $H$ and $\alpha$ values for oil-covered surfaces compared to clean sea surfaces (see, e.g., [6], [56], [60]-[62]). Explanations of this phenomena are often "multiple scattering mechanisms" or departure from the "Bragg scattering" within the oil slicks. In this work, we challenge these explanations by showing that instrument noise can easily account for observed signatures from SNR oil slick surfaces in the $H / \alpha$ space.

Two features that are frequently used in the compactpolarimetric studies (see, e.g., [5], [63]-[67]), namely the degree of polarization $(D o P)$ and the ellipticity angle $(\chi)$ are investigated. Both are calculated from the Stokes vector from a hybrid-polarity SAR system. Reciprocity $\left(S_{H V}=\right.$ $\left.S_{V H}\right)$ is often assumed when calculating the Stokes vector. The influence of system noise might be different in the Stokes vector with and without the reciprocity assumption. To investigate this, we generate two sets of Stokes vectors (reciprocity and non-reciprocity) resulting in two sets of $D_{o P}$ and $\chi$ features. A $D o P=1$ corresponds to fully polarized (fully deterministic) scattered wave, which is equivalent to $H=0$ [68]. Low $D_{o} P$ values has been reported in oil-covered areas [5], [65], [66]. This has been explained by the presence of non-Bragg scattering characterized by "high depolarization" of the backscattered signal from the oil slick surface. The DoP also exhibits poor detection performance using high SNR airborne data [13]. Here we investigate whether system noise is the more likely explanation of these effects, as indicated in [13].

Studies have observed a sign-reversal of the $\chi$ (see, e.g., [66]) for mineral oil slicks. The sign-reversal of the mineral oil slicks was explained in [69] to be caused by different scattering mechanisms between the oil-covered and clean sea surfaces. However, this sign-reversal was only observed in the spaceborne satellite, RS-2, and not in the UAVSAR data. This study investigates whether the system noise is the cause of this "sign-reversal", and not differences in the scattering process between oil and clean sea surfaces. This was also suspected, but not verified in [45].

\section{DATA}

Polarimetric information was acquired from a set of eight RS-2, three TS-X, and three UAVSAR scenes with various types of oil, metocean conditions, range of incidence angles, NESZ values, and signal return from oil slicks and clean sea. The data are radiometrically calibrated and a $9 \times 9$ filter mask is applied to all the scenes when calculating the polarimetric features. When radiometrically calibrating the TS-X data we do not subtract the estimated noise powers in the $\mathrm{HH}$ and VV channels as described in the calibration stage in the TS$\mathrm{X}$ product description [18]. This is because we want to be consistent in comparing the measured scattering vector from all sensors. Table III lists sensor properties, time of acquisition, wind information, incident angle, NESZ, and the estimated MNR used in this study.

All these scenes were acquired during a series of oil-onwater exercises in the North Sea from the years 2011, 2012, 2013, 2015, and 2016, respectively. The wind speeds range from 1.3 to $8 \mathrm{~m} / \mathrm{s}$ (see Table III). The reader is referred to [2], [14], [47] for additional information about these exercises. The data from the UAVSAR and RS-2 used in this study were acquired in the quad-polarimetric (QP) SAR mode, i.e., transmitting and receiving on both the horizontal and vertical polarization channels. The TS-X scenes were acquired with the HH-VV dual-polarimetric SAR mode. The first three TS-X scenes overlap with three of the RS-2 scenes with less than one hour time difference (see [47]). All the scenes capture different concentrations of mineral oil in the oil-water mixtures. The scenes (TS-X and RS-2) from 2011 also contain crude oil.

TABLE III

SENSORS USED, TIME OF ACQUISITION, WIND INFORMATION AROUND ACQUISITION TIME, MEAN INCIDENCE (INC.) ANGLE, NOMINAL MEAN NESZ, AND ESTIMATED MNR. THE QUAD-POLARIMETRIC MODE WAS USED WHEN THE UAVSAR (L-BAND) AND THE RS-2 (C-BAND) SCENES WERE ACQUIRED. ALL THE TS-X (X-BAND) SCENES WERE ACQUIRED IN THE SSC (SINGLE-LOOK COMPLEX) DUAL-POLARIMETRIC (DP) (HH-VV) STRIPMAP MODE. THE WIND INFORMATION IS FROM [47], [70]. TWO MNR VALUES ARE GIVEN FOR RS-2 SINCE THE RANGE AMBIGUITY LEVEL CHANGES DEPENDING ON THE BEAM USED.

\begin{tabular}{l|l|l|l|l|l}
\hline Sensor & $\begin{array}{l}\text { Date } \\
\text { (Time UTC) }\end{array}$ & $\begin{array}{l}\text { Wind } \\
\mathrm{m} / \mathrm{s}\end{array}$ & $\begin{array}{l}\text { Inc. } \\
\text { angle } \\
\left({ }^{\circ}\right.\end{array}$ & $\begin{array}{l}\text { NESZ } \\
(\mathrm{dB})\end{array}$ & $\begin{array}{l}\text { MNR } \\
(\mathrm{dB})\end{array}$ \\
\hline UAVSAR & $09-06-2015(09: 56)$ & 5 & $55-66$ & -44 & \\
UAVSAR & $11-06-2015(08: 46)$ & 8 & $41-44$ & -51 & -16.76 \\
UAVSAR & $11-06-2015(09: 18)$ & 8 & $54-57$ & -45.5 & \\
\hline RS-2 & $08-06-2011(06: 00)$ & $1.6-3.3$ & 47 & -33 & -11.21 \\
RS-2 & $08-06-2011(17: 28)$ & $1.6-3.3$ & 36 & -34 & -11.38 \\
RS-2 & $15-06-2012(06: 20)$ & 4 & 31 & -35 & -11.38 \\
RS-2 & $15-06-2012(17: 49)$ & 3 & 49 & -31 & -11.21 \\
RS-2 & $11-06-2013(17: 20)$ & 5 & 29 & -36 & -11.38 \\
RS-2 & $11-06-2015(17: 27)$ & 6 & 36 & -34 & -11.38 \\
RS-2 & $15-06-2016(06: 07)$ & 7 & 42 & -32 & -11.38 \\
RS-2 & $15-06-2016(17: 36)$ & 8 & 41 & -33 & -11.38 \\
\hline TS-X & $08-06-2011(06: 23)$ & $1.6-3.3$ & 28 & $-23^{a}$ & \\
TS-X & $08-06-2011(17: 12)$ & $1.6-3.3$ & 21 & $-23^{a}$ & -13.88 \\
TS-X & $15-06-2012(17: 29)$ & 3.5 & 41 & $-23^{a}$ & \\
\hline
\end{tabular}

${ }^{a}$ Two NESZ in VV and HH channels and the mean of the two are taken.

ROIs are extracted from each SAR scene. For each oil slick ROI, there is a corresponding clean sea ROI at approximately the same incidence angle. This is done to avoid any incidence angle effects when comparing the oil and clean sea ROIs. 

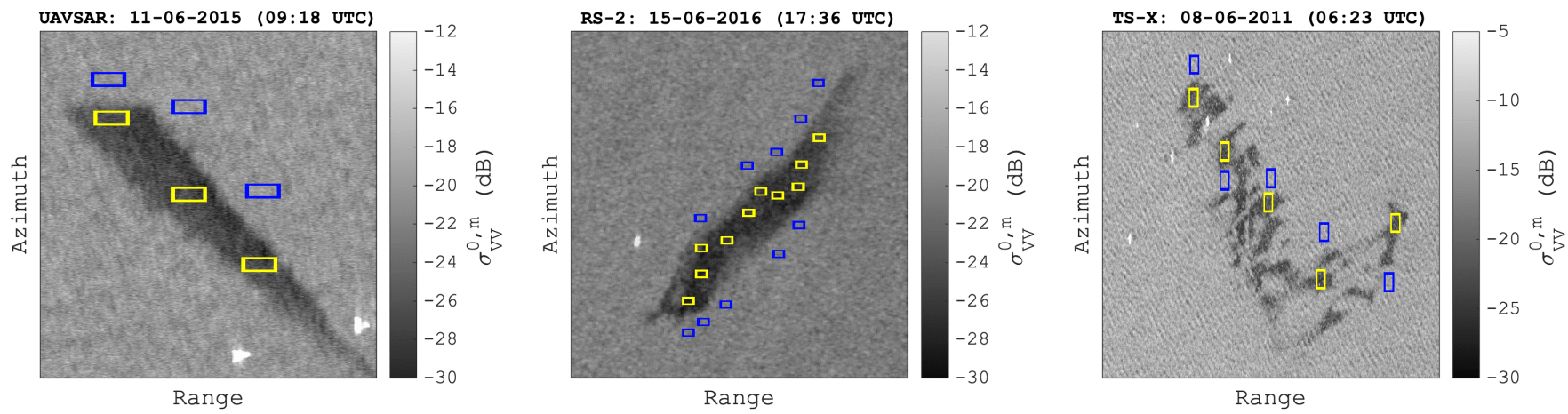

Fig. 1. VV-intensity (in dB) images of UAVSAR, Radarsat-2 (RS-2), and TerraSAR-X (TS-X) covering oil slicks from one of the oil-on-water exercises in the North Sea. The blue ROIs represent clean sea, while the yellow ROIs represents oil-contaminated areas. The scenes are cropped and scaled for display purposes.

There are between 4-10 non-overlapping ROIs within each UAVSAR, RS-2, and TS-X scene. The number of ROIs per scene is constrained by the slick size and our $9 \times 9$ pixel averaging window. Further, the surrounding ships are masked out along with a large portion of the pixels spanning the range and azimuth direction from these ships. This is done to eliminate undesirable sidelobe effects from these bright targets in the scene (see Section II). Second, the same number of pixels (728 corresponding to the size of the smallest ROI) are selected at random within the various sized ROIs. A representative scene and the corresponding ROIs from each of the three sensors is shown in Fig. 1.

The mean measured RCS of a set of pixels randomly selected within these ROIs are displayed in Fig. 2 as a function of $\mathrm{SNR}^{A}$ (signal-to-additive noise ratio) (left panels) and mean incidence angle (right panels). The right panels of Fig. 2 shows lines spanning the 5th and 95th percentiles, the mean intensity (marker), and the nominal NESZ. From the top-left panel in Fig. 2, the separation of the VV backscatter values by frequency (X-, C-, and L-band) is readily seen. For a given surface roughness, $\mathrm{X}$-Band (TS-X) tends to give brighter returns than C-Band (RS-2), which is in turn brighter than the longer wavelength L-Band (UAVSAR). Some VV backscatter data points from both RS-2 and TS-X have mean intensity values close to the noise floor. Whereas some HH backscatter data points from RS-2 have mean intensity values below the noise floor. All the UAVSAR data points from both the $\mathrm{HH}-$ and VV-intensity measurements are well above the noise floor. Most of the HV-intensity values from RS-2 have $\mathrm{SNR}^{A}$ values below $3 \mathrm{~dB}$ and most of the signals from oil and clean sea are below the noise floor (see bottom panels of Fig. 2).

The range of the incidence angles for RS-2 and TS-X spans $20^{\circ}-40^{\circ}$. For UAVSAR, the range of incidence angles is slightly higher at $40^{\circ}-65^{\circ}$. There is a trend of decreasing intensity values as the incidence angle increases in each of the media (oil and clean sea), for RS-2 and TS-X (see the right-most panels of Fig. 2).

The impact of wind is challenging to observe for this study, since the incident angle is not constant across the scenes. In theory, the backscatter increases with wind speed due to the increase in small-scale ocean surface roughness, and the ocean backscatter decreases with increasing incidence angle. For example, more contributions from system noise are expected at low wind speeds measured at high incidence angles. For this study the objective is to observe trends between polarimetric feature values and any contributions from various noise sources in the Bragg scattering region (wind speed in 3-12 m/s and incidence angles in $20^{\circ}-60^{\circ}$ [71]).

Cross-talk between co- and cross-polarization measurements might be significant for some of the UAVSAR measurements. This is because the additive noise floor (NESZ) in UAVSAR data is low $(<-40 \mathrm{~dB})$, and mean VV backscatter values range from $-17 \mathrm{~dB}$ to $-32 \mathrm{~dB}$, whereas the mean $\mathrm{HV}$ backscatter values range from $-35 \mathrm{~dB}$ to $-42 \mathrm{~dB}$, approximately (see Fig. 2). This yields a difference of $>10 \mathrm{~dB}$, and the cross-polarization channel could have contributions from the copolarization channels. For this study, the features based on the cross-polarization are $H, \alpha, D o P$, and $\chi$, which might be particularly exposed to the cross-talk contamination.

\section{Impacts of Multiplicative NOISE IN SNR}

In this study, both the additive and multiplicative noise is used in estimation of the SNR. Common practice is to only calculate the SNR based on the additive noise power, i.e., NESZ. A reason for this is that the NESZ often is available in the product file of various sensors, whereas the multiplicative factors are often left out. Unfortunately, the SNR will be overestimated because of this, and taking proper account of the multiplicative noise components will reduce the SNR values (see, e.g., [34]). Therefore, this section demonstrates the effects of including the multiplicative noise components, namely the ISLR (for all sensors), quantization noise (for RS-2 and UAVSAR), and the ambiguity ratio (for all sensors). The $\mathrm{SNR}^{A}$ (signal-to-additive noise ratio) is estimated using (3). $\mathrm{The} \mathrm{SNR}^{A, M}$ (signal-to-additive and multiplicative noise ratio) is estimated using (4), where the ISLR values (linear units) of each sensor (see Table I) is used and the mean of clean sea pixels within all ROIs in each scene is set to $\sigma^{A V G}$. Fig. 3 shows the relationship between $\mathrm{SNR}^{A}$ and $\mathrm{SNR}^{A, M}$ using the $\mathrm{VV}$ and HH channels, where $\mathrm{SNR}^{A, M}$ is lower than $\mathrm{SNR}^{A}$. With the $\mathrm{SNR}_{V V}^{A}$, all the data points are located above $0 \mathrm{~dB}$, which is no longer the case with the $\mathrm{SNR}_{V V}^{A, M}$, where most of 

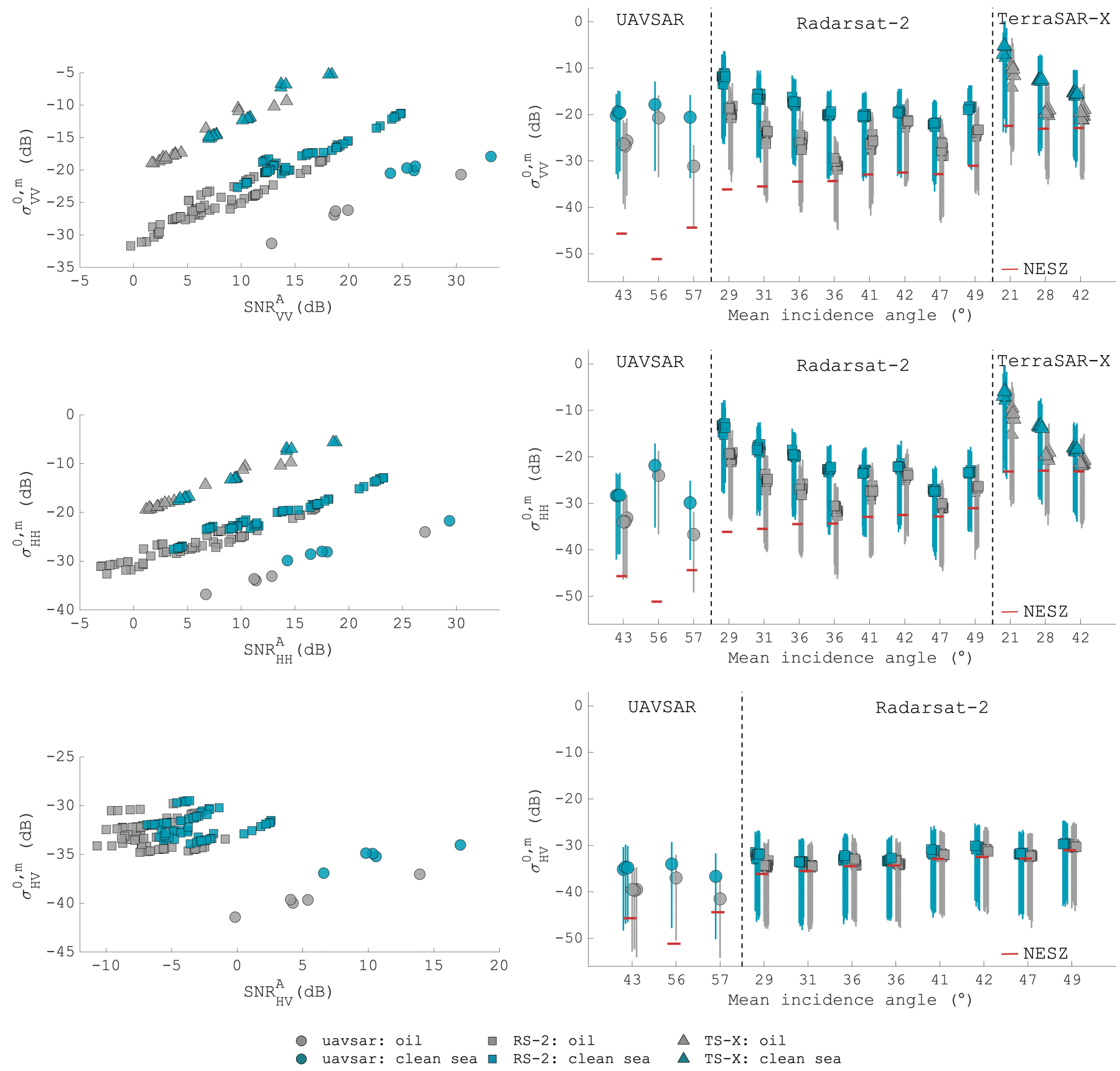

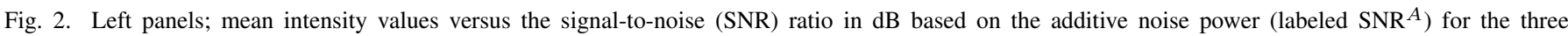
polarization channels (VV, HH, and HV, respectively). Right panels; The 5th, mean intensity, and 95th percentiles for each slick and clean sea region versus the mean incidence angle for each scene. The gray lines are slightly shifted to the left in order to improve the discrimination in the plot. The bottom panels show the HV channel, where there are no ROIs from the TS-X scenes since these were acquired in the HH-VV dual-polarization SAR mode.

the oil data points from RS-2 now have SNR ${ }^{A, M}$ below $0 \mathrm{~dB}$, indicating little signal from the oil slick itself. The oil and clean sea markers for TS-X are located close to the red line indicating that $\mathrm{SNR}^{A}$ and $\mathrm{SNR}^{A, M}$ are very similar. All the markers from the UAVSAR have $\mathrm{SNR}^{A, M}$ values above $10 \mathrm{~dB}$, except for one oil marker that has $\mathrm{SNR}^{A, M}$ around $7 \mathrm{~dB}$.

The multiplicative noise contribution tends to dominate for high SNR ${ }^{A}$ values. For example, the $\mathrm{SNR}_{V V}^{A, M}$ and $\mathrm{SNR}_{H H}^{A, M}$ is on average $11.2 \mathrm{~dB}$ and $5.3 \mathrm{~dB}$ lower than $\mathrm{SNR}_{V V}^{A}$ and $\mathrm{SNR}_{H H}^{A}$ for the oil slick areas in the UAVSAR. For the oil slicks areas in $\mathrm{RS}-2$, the $\mathrm{SNR}_{V V}^{A, M}$ and $\mathrm{SNR}_{H H}^{A, M}$ is on average $8.7 \mathrm{~dB}$ and $6.7 \mathrm{~dB}$ lower than $\mathrm{SNR}_{V V}^{A}$ and $\mathrm{SNR}_{H H}^{A}$. Finally, for the oil slicks areas in TS-X, the $\mathrm{SNR}_{V V}^{A, M}$ and $\mathrm{SNR}_{H H}^{A, M}$ is on average $2.8 \mathrm{~dB}$ and $2.5 \mathrm{~dB}$ lower than $\mathrm{SNR}_{V V}^{A}$ and $\mathrm{SNR}_{H H}^{A}$.

\section{RESUlTS AND DisCUSSION}

This section is divided into three subsections reflecting the objectives presented in Section I. Subsection (1) presents and discusses the feature sensitivity to the additive and multiplicative noise power. Subsection (2) demonstrates how the noise 


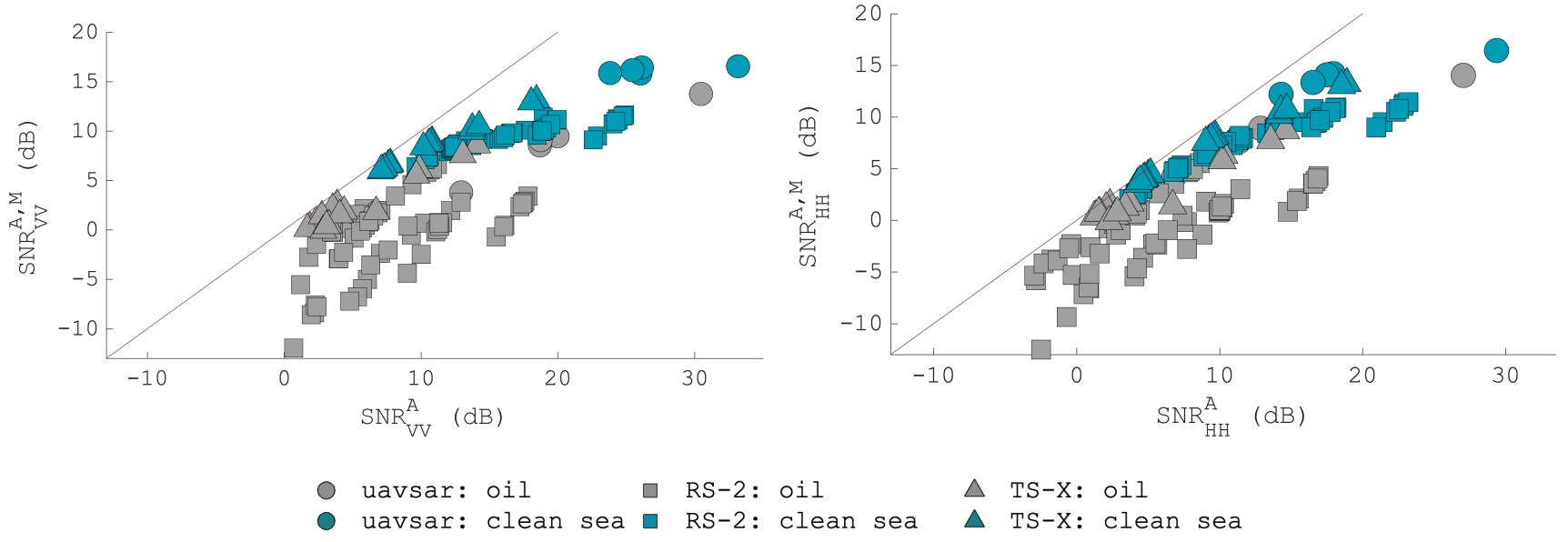

Fig. 3. Relationship between the mean $\mathrm{SNR}^{A}$ (signal-to-additive noise ratio) versus the mean $\mathrm{SNR}^{A, M}$ (signal-to-additive and multiplicative noise ratio) for the UAVSAR, RS-2, and TS-X ROIs. The red line is where $\mathrm{SNR}^{A}$ and $\mathrm{SNR}^{A, M}$ are equal.

affects the $H / \alpha$ decomposition, and how the SNR threshold is identified. Finally, subsection (3) illustrates how each of the different features behaves when the additive noise power is subtracted.

\section{(1) Feature sensitivity to additive and multiplicative noise}

We aim to provide an understanding of the sensitivity to both the additive and multiplicative noise for the set of features investigated, and to further highlight which features are strongly affected by the noise for the two investigated media (oil and clean sea). Figs. 4-7 show the mean of a given feature for a set of ROIs plotted against the $\mathrm{SNR}_{H H}^{A}$ (left panels) and $\mathrm{SNR}_{H H}^{A, M}$ (right panels) in $\mathrm{dB}$. The continuous lines show the results of our simulations of adding noise to the high-SNR UAVSAR data (see Appendix C). The simulated noise power plots are not shown for $P D$ and $r_{C O}$, which is due to the fact that the simulated noise powers cancels in the calculation of $P D$ and decorrelates in $r_{C O}$. For RS-2 and TS-X the $\operatorname{SNR}_{H H}^{A}$ is calculated from the RCS and the NESZ given in the product file, and for the UAVSAR the 5th order polynomial with updated coefficients are used (similar to [26]). The $\mathrm{SNR}_{H H}^{A, M}$ is calculated from (4) with MNR given in Table III. Because both $\mathrm{SNR}_{H H}^{A}$ and $\mathrm{SNR}_{H H}^{A, M}$ are functions of $\sigma_{H H}^{0, m}$, the values plotted are the mean for the same set of pixels randomly selected within each ROI.

\section{Polarization difference $(P D)$}

In the left panels of Fig. 4, lower $P D$ values of the oilcovered areas compared to the clean sea regions can be seen. This observation corroborates previous findings [47]. The reduction in $P D$ due to the presence of oil is most likely caused by the dampening of the ocean surface roughness [48]. The $P D$ is expected to be close to zero for pure random noise, assuming the noise in the $\mathrm{HH}$ and VV channels are similar. A slight trend of increasing $P D$ values with $\mathrm{SNR}_{H H}^{A}$ and $\mathrm{SNR}_{H H}^{A, M}$ for all the three sensors is observed, especially for the oil markers. However, there is a wide spread in the
$P D$ values across the $\mathrm{SNR}_{H H}^{A}$ which might be interpreted as sensitivity to the oil properties and metocean conditions, that vary across the scenes used in this analysis. However, less spreading is observed for the $\mathrm{SNR}_{H H}^{A, M}$, which are more consistent with a simple, downward linear trend of decreasing $P D$ with decreasing SNR.

In conclusion, the downward trend in $P D$ due to the presence of oil is entirely consistent with a Bragg scatter model for which the VV backscatter is greater than the HH (the high SNR case), with increasing levels of noise added for which the $\mathrm{HH}$ and $\mathrm{VV}$ expected values are the same, and therefore $P D \rightarrow 0$ (the low SNR case).

\section{Real part of the copolarization cross product $\left(r_{C O}\right)$}

The oil has lower $r_{C O}$ (real part of the copolarization cross product) values than the clean sea (right panels of Fig. 4). Again, this matches previous findings [2], [47], [72]. The authors of [72] explained this observation as the presence of a non-Bragg scattering process within the oil slick. However, our $\mathrm{SNR}_{H H}^{A, M}$ results show a simple downward linear trend in $r_{C O}$ as SNR decreases. The reduction of the $r_{C O}$ values in the presence of oil is therefore most likely related to the low backscattering return from the oil slick, resulting in high influence of the decorrelation effects from system noise. The expected value of $r_{C O}$ for pure random noise is $0 . r_{C O}$ values are high at lower incidence angle, where less noise is expected, for all three sensors. At high incidence angle (large markers in the right panels of Fig. 4) the $r_{C O}$ is low.

For $r_{C O}$, the results for different radar frequencies are differentiated at higher SNR; these results indicate that for this particular parameter, $\mathrm{X}$-band is more sensitive to oil characteristics than $\mathrm{C}$-band, which is more sensitive than Lband. No other parameter shows this clear separation between all three bands.

\section{Copolarization ratio $\left(\gamma_{H H / V V}\right)$}

Applying a tilted Bragg model, the $\gamma_{H H / V V}$ values from the oil slick areas are expected to be slightly larger than for 

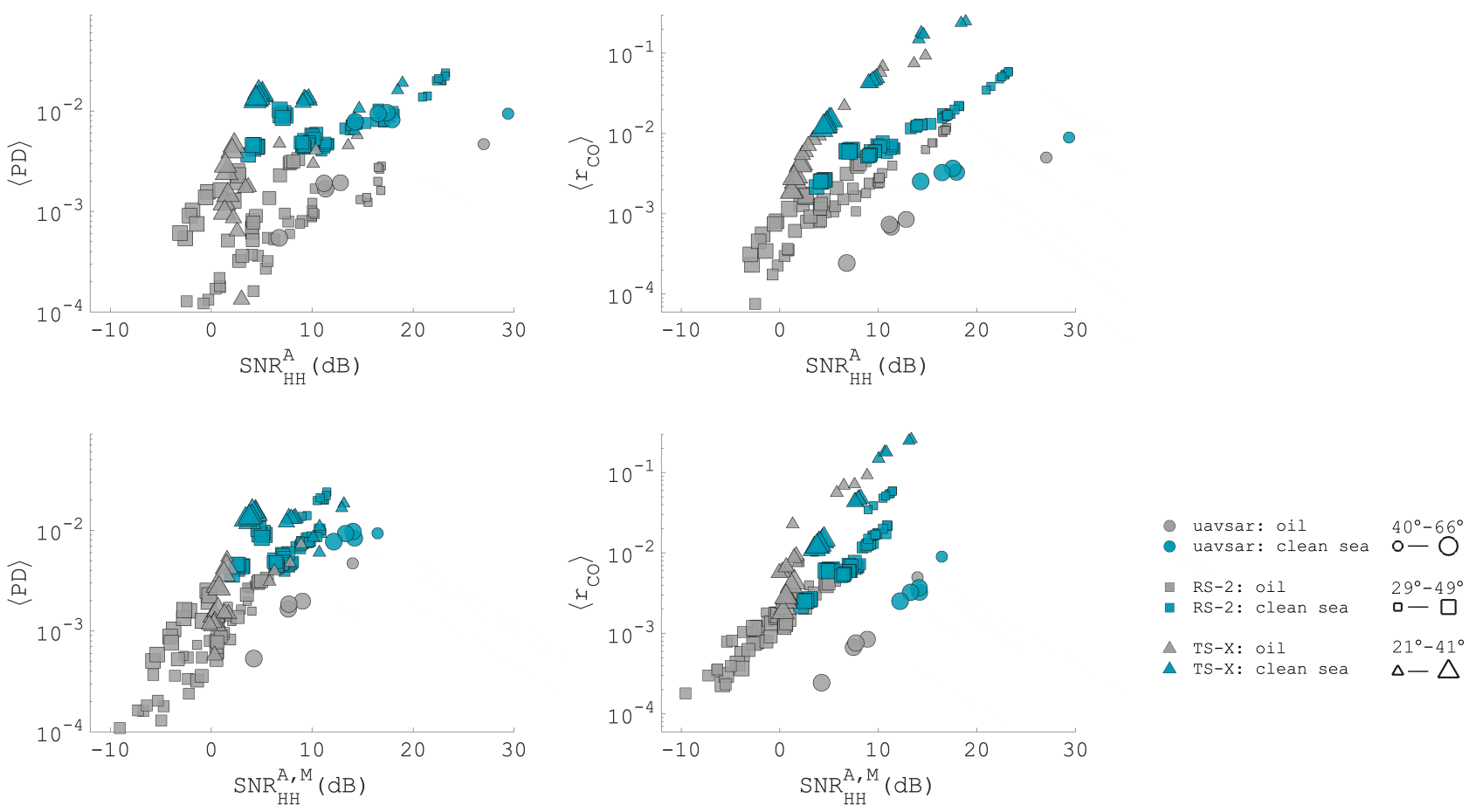

Fig. 4. Top: mean of $P D$ and $r_{C O}$ versus $\mathrm{SNR}_{H H}^{A}(\mathrm{~dB})$. Bottom: mean of $P D$ and $r_{C O}$ versus $\mathrm{SNR}_{H H}^{A, M}(\mathrm{~dB})$. A log-scale is applied on all y-axis to better illustrate the trend. The expected $P D$ and $r_{C O}$ of pure noise is 0 in linear units.

the clean sea areas, depending on the concentration of oil on the surface [10]. This fits our high SNR observations in the left panels of Fig. 5. For pure random noise, $\gamma_{H H / V V}$ is close to 1 (see Table IV), as illustrated with the "N" symbol in Fig. 5. For the clean sea results, larger sized markers, indicating a higher incidence angle, are located below the smaller markers for each sensor, confirming that $\gamma_{H H / V V}$ decreases as a function of increasing incidence angle (see, e.g., [10], [12]). Similar to the $P D$ results, we again see a wide spread in the oil slick $\gamma_{H H / V V}$ values across the $\mathrm{SNR}_{H H}^{A}$ which can be interpreted as sensitivity to the oil properties and metocean conditions. Again the large spread in values for clean ocean even at high SNR, a strong dependence on metocean conditions is likely consistent with [12]. But less spreading is observed for the $\mathrm{SNR}_{H H}^{A, M}$ results and, in general, $\gamma_{H H / V V}$ increases with decreasing $\operatorname{SNR}_{H H}^{A}$ and $\operatorname{SNR}_{H H}^{A, M}$. This trend matches well with our simulations of adding increasing levels of noise to the UAVSAR (continuous lines), and corroborates the observations of Minchew et al. [10] at high incidence angles. Here the increasing dominance of noise over backscatter explains the $\gamma_{H H / V V}$ observations, with this feature eventually approaching the value 1 with increasing noise power.

\section{Magnitude of the copolarization correlation coefficient ( $\left.\rho_{C O}\right)$}

According to the literature, the $\rho_{C O}$ feature tends to generate higher values for clean sea compared to oil-covered surfaces (see Section IV). The center panels of Fig. 5 supports this, as most of the clean sea markers are above the oil markers. If the measured signal is heavily contaminated by noise, the expected $\rho_{C O}$ value is 0 , as indicated by the "N" symbol in Fig. 5. Adding noise to the UAVSAR data results in decreasing $\rho_{C O}$ values as both the $\operatorname{SNR}_{H H}^{A}$ and $\operatorname{SNR}_{H H}^{A, M}$ decreases. The spaceborne markers (squares and triangles) align well with the UAVSAR simulations, once all the noise sources are properly accounted for (right-hand panel). This was also pointed out in [13], where the instrumental noise induced a decorrelation effect between the $\mathrm{HH}$ and VV. Note that $\rho_{C O}$ is not exactly 1 for the high-SNR clean sea UAVSAR data; for which $S N R_{H H}^{A}=30 \mathrm{~dB}$ and $S N R_{H H}^{A, M}=18 \mathrm{~dB}$, which can be explained by the presence of multiplicative noise (which lowers the effective SNR) once that is taken into account (right-hand panel). The reduction in $\rho_{C O}$ for the oil in all cases is most likely due to system noise and not to exotic scattering properties within the oil.

\section{Standard deviation of the copolarization phase difference $\left(\sigma_{\phi_{C O}}\right)$}

As shown in right panels of Fig. 5, $\sigma_{\phi_{C O}}$ has lower values for clean sea than for oil slicks. This observation has also been reported in previous studies (see, e.g., [54]-[57]). The $\sigma_{\phi_{C O}}$ is uniformly distributed, and the expected value of $\sigma_{\phi_{C O}}$ for pure Gaussian noise is 1.81 (see Table IV Appendix A). The $\sigma_{\phi_{C O}}$ values tend to increase with decreasing $\operatorname{SNR}_{H H}^{A}$ and $\mathrm{SNR}_{H H}^{A, M}$ values (see center panels of Fig. 5), consistent with our simulations adding noise to the UAVSAR results. 

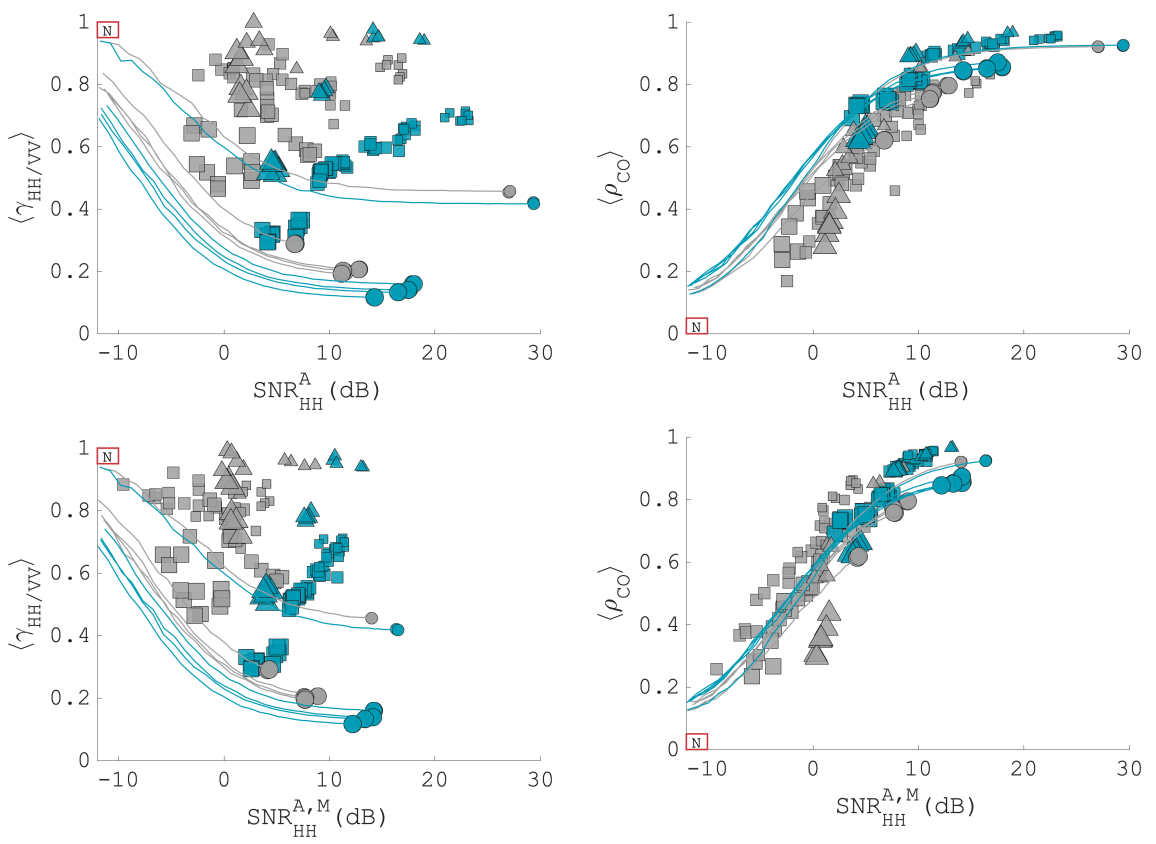

uavsar: oil $40^{\circ}-66^{\circ}$

- uavsar: clean sea $0-\bigcirc$

RS-2: clean sea

$29^{\circ}-49^{\circ}$

$\triangle \mathrm{TS}-\mathrm{X}$ : oil

AS-X: clean sea

$\Delta-\triangle$
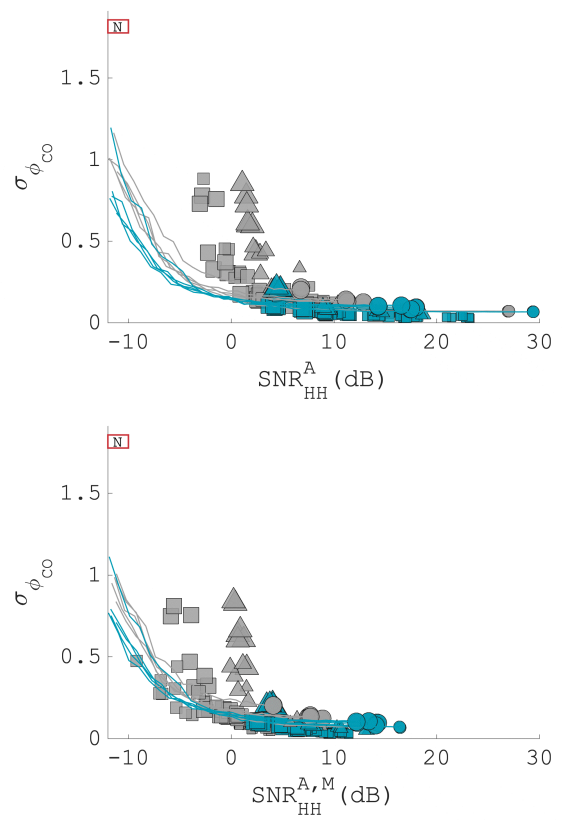

Fig. 5. Top: mean of $\gamma_{H H / V V}$, mean of $\rho_{C O}$, and standard deviation of $\phi_{C O}$ versus $\mathrm{SNR}_{H H}^{A}(\mathrm{~dB})$. Bottom: mean of $\gamma_{H H / V V}$, mean of $\rho_{C O}$, and standard deviation of $\phi_{C O}$ versus $\mathrm{SNR}_{H H}^{A, M}(\mathrm{~dB})$. The circular markers are the mean values of the ROIs from the UAVSAR data, while grey and blue continuous lines represent the degree of simulated noise power added to the UAVSAR. The legend of incidence angle ranges is on the bottom. The box with "N" is the expected feature value of Gaussian white noise (see Table IV in Appendix A).

As pointed out in [45] and confirmed by Fig. 5, this extreme broadening of the HH-VV phase difference distribution is most likely due to instrumental noise rather than a different type of scattering within the oil slicks.

\section{Entropy $\left(H_{C O}\right.$ and $\left.H\right)$}

Higher entropy values (both $H$ and $H_{C O}$ ) are observed for the oil than the clean sea, which matches previous findings (see, e.g., [6], [56], [60]-[62]). However, this difference is mainly due to higher noise contribution in the measured oil slick signal compared to the signal from the clean sea. The left and center panels of Fig. 6 support this, where both $H$ and $H_{C O}$ increase with decreasing $\operatorname{SNR}_{H H}^{A}$ and $\operatorname{SNR}_{H H}^{A, M}$, following the trend lines for the simulated noise added to UAVSAR data. The correspondence is more marked in the $\mathrm{SNR}_{H H}^{A, M}$ results. The high entropy is clearly related to the high noise power in the data, and not differences in scattering processes between oil and clean sea.

\section{Mean alpha angle $(\alpha)$}

Higher $\alpha$ values are observed for the oil than for the clean sea. This aligns with previous findings, but the more likely explanation for higher $\alpha$ values is higher noise power, rather than different scattering properties between the clean sea and the oil. Complex Gaussian white noise will have a mean $\alpha$ value of $60^{\circ}$. The observations of $\alpha$ shown in the right panels of Fig. 6 increase towards $60^{\circ}$ as the $\mathrm{SNR}_{H H}^{A}$ and $\mathrm{SNR}_{H H}^{A, M}$ decreases. Again, the $\mathrm{SNR}_{H H}^{A, M}$ results fit better with the simulated noise at lower SNR values, compared to $\mathrm{SNR}_{H H}^{A}$. The discussion on the $H$ and $\alpha$ continues in subsection (2), where the noise effects on the $H / \alpha$ space are investigated.

\section{Degree of polarization (DoP)}

In the left panels of Fig. 7, the color-filled squares and circles for each class (oil and clean sea) represent the nonreciprocity case, and the $D o P$ values for which reciprocity is assumed are indicated by non-filled boxes and circles. The continuous lines represent the noise added to the UAVSAR data when reciprocity is not assumed, while the dashed lines are for the scenario in which reciprocity is assumed. There is a clear difference between the clean sea and the oil markers, where lower $D o P$ values of the oil are observed for both the reciprocity and the non-reciprocity case. The expected value of $D_{o} P$ for Gaussian white noise is 0 for non-reciprocity and 0.5 with the reciprocity assumption. In general, the $D o P$ increases with increasing $\mathrm{SNR}_{H H}^{A}$ and $\mathrm{SNR}_{H H}^{A, M}$ (see left panels of Fig. 7). As pointed out in [68], the $D o P$ feature is similar to $1-H$, where low $D o P$ indicates high depolarization. The $D o P$ has been used to measure the departure from Bragg, and as shown here, the observed departure from $\operatorname{Bragg}(D o P \approx 1)$ occurs at low SNR. At low $\operatorname{SNR}_{H H}^{A}$ and $\operatorname{SNR}_{H H}^{A, M}$ the $D o P$ 

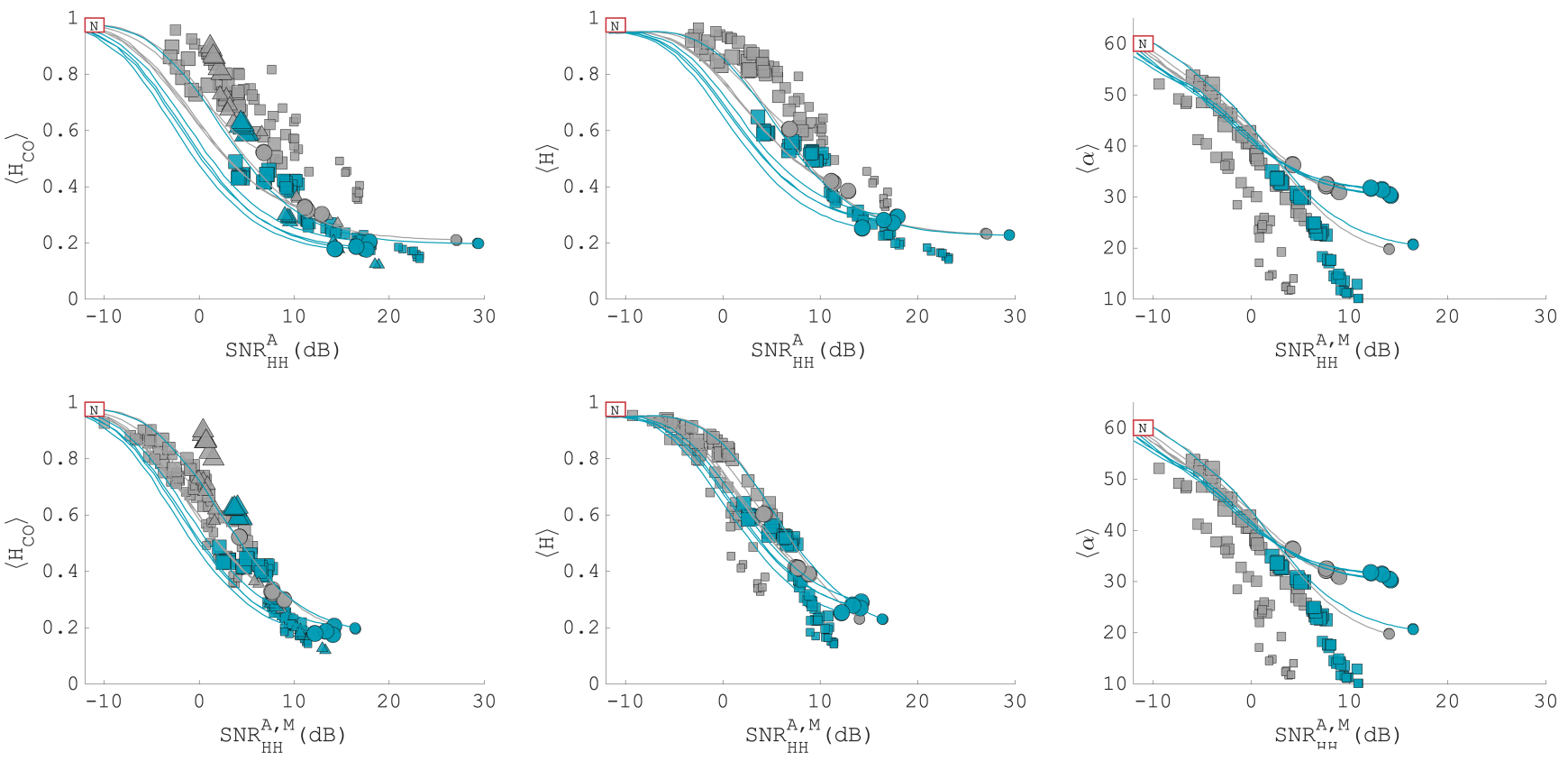

- uavsar: oil

uavsar: oil $40^{\circ}-66^{\circ} \square \mathrm{RS}-2$ : oil
uavsar: clean sea $0-\bigcirc-2$ clean sea

$29^{\circ}-49^{\circ} \triangle$ TS-X: oil
$\square-\square \quad$ TS-X: clean sea

$21^{\circ}-41^{\circ}$

- uavsar: simulated noise (oil)

Fig. 6. Top: mean of $H_{C O}, H$, and $\alpha$ versus $\mathrm{SNR}_{H H}^{A}(\mathrm{~dB})$. Bottom: mean of $H_{C O}, H$, and $\alpha$ versus $\mathrm{SNR}{ }_{H}^{A}, M(\mathrm{~dB})$. The circular markers are the mean values of the ROIs from the UAVSAR data, while grey and blue continuous lines represent the degree of simulated noise power added to the UAVSAR data. The legend of incidence angle ranges is given on the bottom. The box with "N" is the expected feature value of Gaussian white noise (see Table IV in Appendix A).
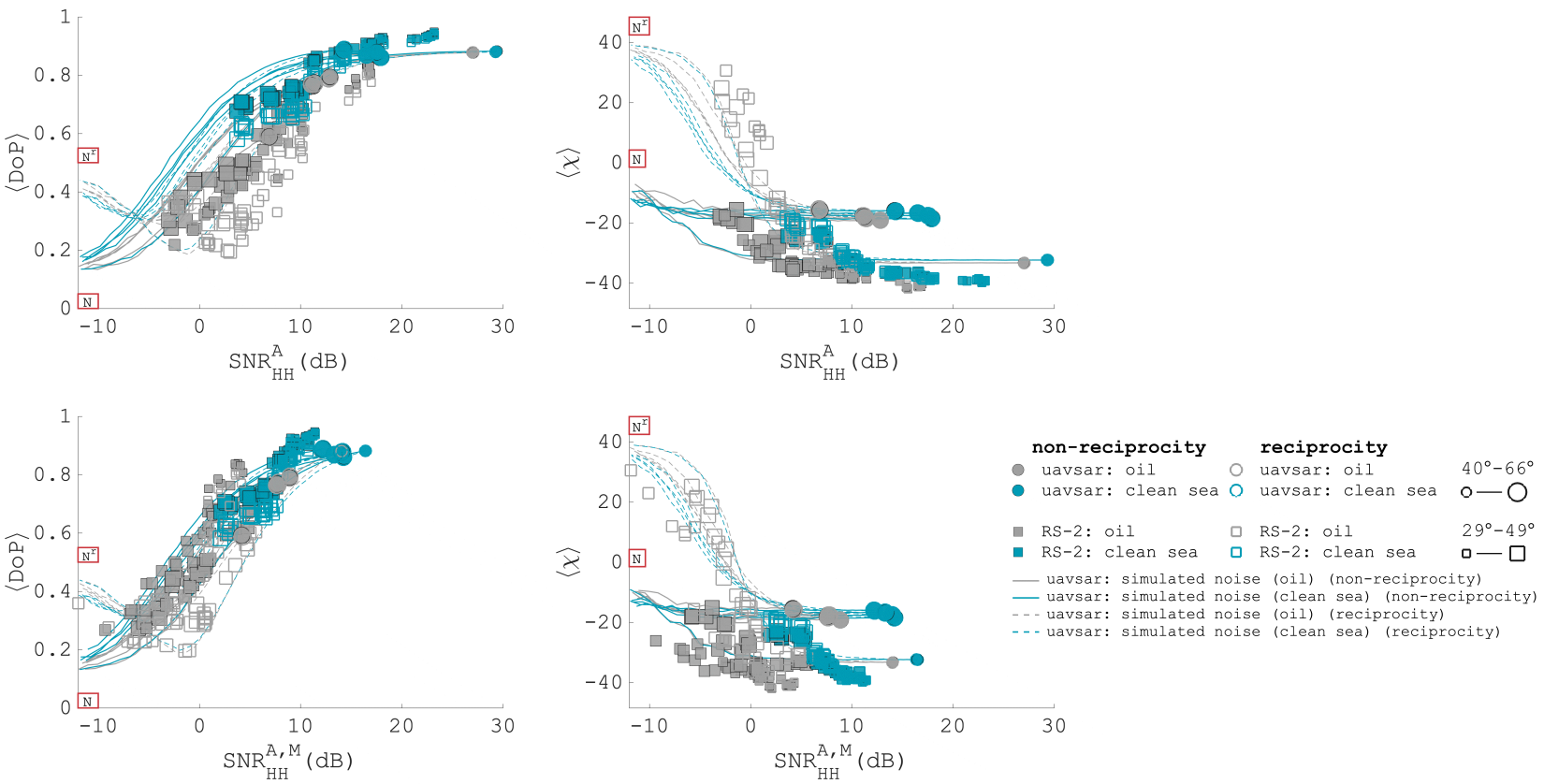

Fig. 7. Top: mean of $D o P$ and $\chi$ versus $\mathrm{SNR}_{H H}^{A}(\mathrm{~dB})$. Bottom: mean of $D o P$ and $\chi$ versus $\mathrm{SNR}_{H H}^{A, M}(\mathrm{~dB})$. The circular markers are the mean values of the ROIs from the UAVSAR data, while grey and blue continuous (dashed) lines represent the degree of simulated noise power added to the UAVSAR data for the non-reciprocity (reciprocity) case. The boxes with " ${ }^{r}$ " and " $\mathrm{N}$ " are the expected feature value of Gaussian white noise for reciprocity and non-reciprocity cases (see Table IV in Appendix A). 
starts to increase for the reciprocity-assumed case, which is a result of the extra noise component in $S_{3}$ of the $D o P$ expression (see Appendix B). Again, the $\operatorname{SNR}_{H H}^{A, M}$ fits better with the simulated noise compared to $\mathrm{SNR}_{H H}^{A}$. The strong correlation of the $D_{o P}$ values with the $\mathrm{SNR}_{H H}^{A}$ in Fig. 7 matches the observation in [13].

\section{Ellipticity angle $(\chi)$}

Like the $D_{o P}$, the $\chi$ is calculated from two Stokes vectors (reciprocity and non-reciprocity assumed case). Few differences between the oil and the clean sea markers can be seen for the non-reciprocity case in the right panels of Fig. 7. As the noise increases the mean of the $\chi$ approaches the value 0 . In general, $\chi$ is always positive for the non-reciprocity case, but a decreasing trend of the $\chi$ as the $\operatorname{SNR}_{H H}^{A}$ and $\operatorname{SNR}_{H H}^{A, M}$ increases is identified. However, for the reciprocity case, the $\chi$ of both the real and simulated noise have a more rapid decrease with increasing $\mathrm{SNR}_{H H}^{A}$ and $\mathrm{SNR}_{H H}^{A, M}$. Again, the $\mathrm{SNR}_{H H}^{A, M}$ fits the simulated noise better. This is easily explained by the extra noise component in $S_{3}$, for which a clear sign-reversal is identified when the noise power increases. The only factor contributing to the sign-reversal is the $S_{3}$ parameter since this is the only component in $\chi$ that can either be negative or positive (see Appendix B). $\chi$ is also affected by the reciprocity assumption, especially at low SNR. Therefore, the presence of a sign-reversal in $\chi$ for oil spill data in polarimetric SAR is accounted for by noise.

\section{(2) Noise in the $H / \alpha$ decomposition}

The motivation behind this section is to investigate the behavior of the $H / \alpha$ space as a function of both the $\mathrm{SNR}^{A}$ and $\mathrm{SNR}^{A, M}$ using only real data from RS-2 and UAVSAR. The $H$ and $\alpha$ are both calculated from the $3 \times 3$ sample coherency matrix using a $9 \times 9$ averaging filter mask.

The ocean backscatter is dominated by resonance scattering also known as Bragg scattering, within the SAR incidence angles $20^{\circ}-60^{\circ}$ (see, e.g., [71]), and for low to moderate wind $3-12 \mathrm{~m} / \mathrm{s}$. The $H / \alpha$ space has been used frequently to interpret the scattering properties (see, e.g., [6], [10], [56], [60]-[62]). Minchew et al. [10] observed Bragg scattering within the oil slick region using UAVSAR data. The authors concluded that the departure from the Bragg region within the oil slick was mainly due to the instrumental noise, which is verified here based on Fig. 8. The Bragg scattering region (see [59]) is defined within the black boxes in Fig. 8 and the markers are colored based on their $\mathrm{SNR}_{H H}^{A}$ (top panels) in dB. Similar results applies for $\mathrm{SNR}_{H H}^{A}$, which is not shown, but the SNR values are higher with VV compared with $\mathrm{HH}$. Each marker represents one pixel from the set of 728 pixels that are randomly selected within each ROI.

The majority of the oil markers fall outside the Bragg region due to the high level of noise. This confirms the observations in [10] and the discussion in [45]. There is a remarkable correlation between increasing $\alpha$ and $H$ values as the $\mathrm{SNR}^{A}$ decreases, and a decrease in $\mathrm{SNR}^{A}$ results in a clear departure from the Bragg region. The clean sea markers are, as expected, mostly located within the Bragg region. But even here, when the additive noise power increases some markers fall outside this region.

The center and bottom panels in Fig. 8 show blue and red histograms, that are calculated based on $\mathrm{SNR}_{H H}^{A}$ and $\mathrm{SNR}_{H H}^{A, M}$ values originated from the Bragg scattering region (blue) and outside this region (red). These panels contain information about the SNR threshold that is recommended before the data is too contaminated by the additive and multiplicative noise for any meaningful polarimetric scattering analysis.

The peak overlap within the blue and red histograms is around $S N R_{H H}^{A}=10 \mathrm{~dB}$. Considering the bottom panels of Fig. 8, this peak overlap within the red and blue histograms varies between $0-8 \mathrm{~dB}$ for the $\mathrm{SNR}_{H H}^{A, M}$, where no clear threshold is observed. The oil markers inside the Bragg region in the top panel of Fig. 8 indicate a higher signal return compared to the oil indicated by markers outside this region. Unfortunately, the contribution from the multiplicative noise sources is high inside the Bragg region despite the high $\mathrm{SNR}_{H H}^{A}$ for the oil (larger than 10dB). In the bottom-left panel of Fig. 8, the mean and standard deviation of the $\mathrm{SNR}_{H H}^{A, M}$ inside the box (Bragg region) are $3.2 \pm 1.8 \mathrm{~dB}$. Since the $H / \alpha$ is extremely sensitive to the presence of noise, we conclude that the $H / \alpha$ is not recommended for extracting information about the scattering properties within oil slicks, at least with existing sensors that do not have much higher SNR for oil returns.

\section{(3) The impact of subtracting the additive noise}

This section shows the effect of subtracting the additive noise from the covariance/coherency matrix prior to calculating the features, and whether this procedure can produce reliable feature value of low-backscattered targets like the oil slick as studied here. Only scenes from RS-2 are used. In this part of the study, we only consider the additive noise component (not the multiplicative noise factor) since it is unclear how the multiplicative noise impacts the off-diagonal elements in the coherency matrix, and subtracting the additive noise is a common procedure in the literature [10], [73][76]. The noise cannot be removed from the complex target scattering matrix, but previous studies have subtracted the noise power from the diagonal elements in the second order sample covariance matrix (see, e.g., [73]-[76]). The features investigated in this work can all be estimated from either the sample covariance or the coherency matrix, allowing us to evaluate the effect of the noise subtraction. The nominal NESZ provided in the RS-2 product file of each scene is used when subtracting the noise power from the sample covariance/coherency matrix. This is because the estimated NESZ values using the method suggested in [75] and [76] shows close to the same values as the nominal NESZ (see Appendix D).

Fig. 9 shows the feature sensitivity to the noise subtraction using only the RS-2 ROIs from both the oil-covered (squares) and the clean sea (diamonds) surfaces. Noise subtraction can only be performed for the set of features that are a function of one or more intensity components. The $r_{C O}$ and $P D$ are left out in Fig. 9, since noise subtraction will not have any effect in these two features. Recall, $r_{C O}$ is the real part of the 
correlation between the complex $\mathrm{HH}$ and VV measurements. The complex noise components decorrelate with each other and to the signal (see (7)), and $r_{C O}$ is therefore not a function of the noise power $\left(\sigma^{n}\right)$. The $P D$ is the difference between the $\mathrm{VV}$ and $\mathrm{HH}$ intensities, and only one noise power in $\mathrm{HH}$ and VV channels are provided and assumed equal. Hence, the two noise components in $P D$ cancel.

All the features shown in Fig. 9 are affected by the noise subtraction. In general, the difference between the mean feature value between the original and the noise subtracted feature increases with the noise, i.e., $S N R_{H H}$ decreases.

Recall from the previous section, that the $\gamma_{H H / V V}$ tends towards the value 1 as the noise power increases. Having a decrease in $\gamma_{H H / V V}$ after noise subtraction indicates that noise power contributed significantly to a higher $\gamma_{H H / V V}$ value, especially for the oil markers. The noise subtraction for the oil markers has a larger effect than for the clean sea markers, which is due to higher SNR for the clean sea than for the oil slick. Hence, the $\gamma_{H H / V V}$ values are more similar between the oil and clean sea after the noise subtraction.

For Gaussian white noise, the $\rho_{C O}$ is 0 . Hence, subtracting the noise should increase the $\rho_{C O}$ values, which is clearly demonstrated in the top-center panel of Fig. 9. As pointed out in Section IV, previous studies found low $\rho_{C O}$ for oil-covered areas, and this was explained by the different scattering properties between clean sea and oil-covered surfaces. Here, subtracting the noise resulted in similar $\rho_{C O}$ values between the two classes, indicating that the variation in $\rho_{C O}$ between the oil and the clean sea is most likely due to the additive noise.

Further, a decrease in both $H_{C O}$ and $H$ as a result of the noise subtraction is also demonstrated in the top-right and center-left panels of Fig. 9. This is expected as random noise contributes to high entropy values. However, there is still a trend with increasing entropy (both $H_{C O}$ and $H$ ) as the $S N R_{H H}$ decreases after noise subtraction. Since intermediate entropy values are expected also for oil-covered regions, subtracting the noise results in a more reasonable entropy value for the oil markers.

The $\alpha$ increases with the noise power for both the original and the noise subtracted markers. For Gaussian white noise, the $\alpha$ is expected to be in the area around $60^{\circ}$ (see Table IV Appendix A). Based on the differences between the dashed and continuous lines, the $\alpha$ values after noise subtraction have decreased, especially for low SNR. The majority of the markers seems to be below $\alpha=42.5^{\circ}$, which is the upper threshold of the Bragg scattering region. We conclude that the noise subtraction has an effect on the $\alpha$.
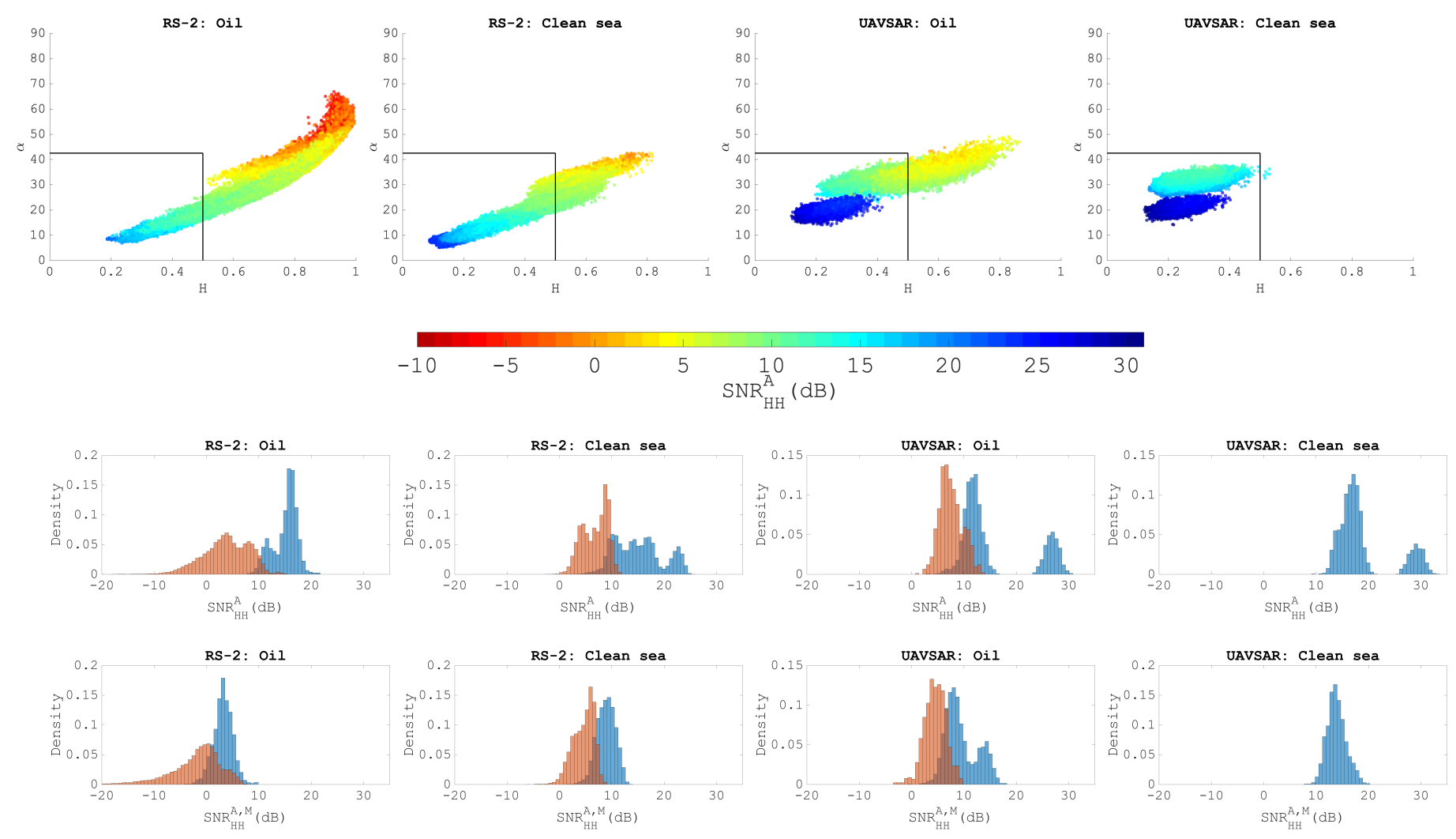

Outside Bragg scattering

Inside Bragg scattering

Fig. 8. H/ $\alpha$ plots of RS-2 and the UAVSAR data containing clean sea and oil slicks. The difference in, e.g., incidence angle and metocean conditions might cause the difference between the colored markers within each panel. Top: the markers are colored based on the SNR $H_{H}^{A}$ level. Center: histograms of the $\mathrm{SNR}_{H H}^{A}$ values inside and outside the Bragg scattering region. Bottom: histograms of the $\mathrm{SNR}_{H H}^{A, M}$ values inside and outside the Bragg scattering region. 

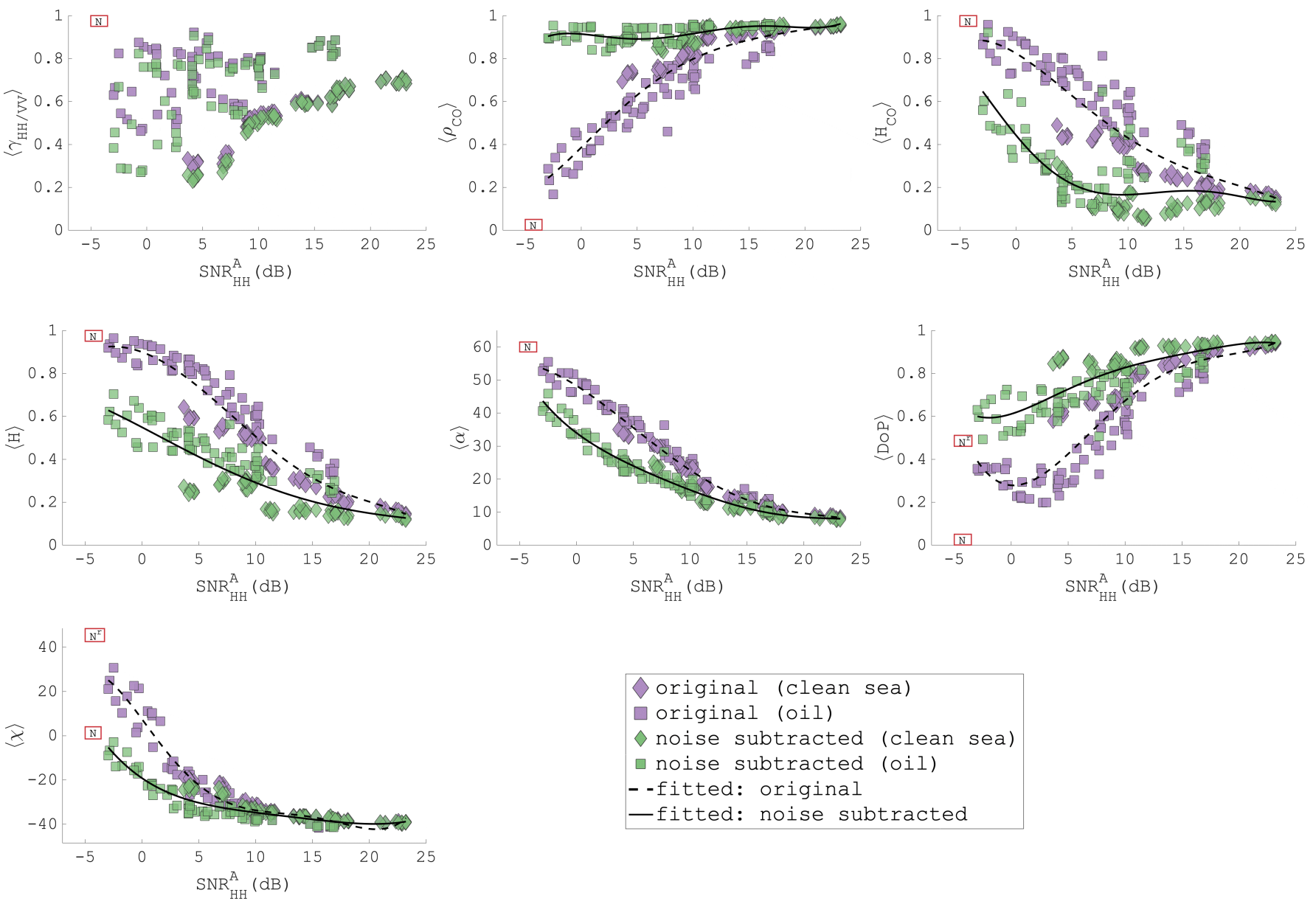

Fig. 9. The effect of noise subtraction on the mean feature value (of both clean sea (diamond) and oil (square) ROIs) versus the $S N R_{H H}$ (dB) using RS-2 data. The box with the "N" symbol is the expected feature value of pure random noise. The $D o P$ and $\chi$ are from the reciprocity-assumed Stokes vector, but both "N" ${ }^{r}$ " and "N" is shown.

Subtracting the noise resulted in similar $D o P$ values between clean sea and oil, and higher values after the noise subtraction are identified (center-right panel of Fig. 9). This is expected as the noise will generate lower $D o P$ values. In this panel, only the reciprocity-assumed $D o P$ is used. The $D o P$ is a child parameter from the Stokes vector, where the Stokes vector is calculated using the method presented in [63], which is based on the elements from the $3 \times 3$ covariance matrix, from which the noise is subtracted. After the noise subtraction the oil markers are almost aligned with the clean sea markers. Hence, the $D o P$ shows a poorer contrast between the oil and the clean sea after the noise subtraction, and any oil/sea contrast seen in this feature can be explained by noise. As demonstrated in the previous section, the $\chi$ changed sign when the noise increased. A sign reversal is no longer visible after the noise subtraction, and most of the $\chi$ values are negative after this procedure. Thus, this feature becomes useless for oil spill detection due to similar values between the clean sea and oil markers.

\section{CONCLUSIONS}

The system noise in SAR data will have an influence on the interpretation of the scattering properties, particularly for low backscatter targets like oil slicks. Mischaracterizations can arise if all of the various noise sources (see Sections II and III) are not considered. Oil spill detection is still possible for noisy data, simply due to the marked contrast in the backscatter levels. This study explored the limitation of satellite SARs to perform reliable oil spill characterization using scattering properties from polarimetric features. Additionally, it may also be possible to identify regions of thicker oil within a slick, since thicker oil may have lower backscatter returns. This is due to higher damping of the small capillary and gravity waves, and noise thus contributes a larger fraction of the measured signal than for thinner oil films.

This study shows the impact of including both additive and multiplicative noise factors in the estimation of the SNR. From spaceborne sensors such as RS-2 and TS-X, the majority of the measured signal is comprised of noise originating from various sources, dominating the backscattered signal from the 
oil itself. This is demonstrated by $\mathrm{SNR}^{A, M}$ having values close to or even less than $\mathrm{OdB}$. However, this is not the case for the UAVSAR, which has much higher SNR.

All the features investigated are influenced by both additive and multiplicative noise, some more than others. However, the $P D, \gamma_{H H / V V}$ and $r_{C O}$ had highest spread in their feature values as a function of the $\mathrm{SNR}^{A}$, which was reduced significantly for $\mathrm{SNR}^{A, M}$. The remaining features $\left(\rho_{C O}, H\right.$, $\left.H_{C O}, \alpha, D o P, \chi\right)$ simulated by adding noise to UAVSAR data show a similar trend with the corresponding feature extracted from TS-X and RS-2 data. This trend is observed at different incidence angles and at low to moderate wind speed range (3-8 $\mathrm{m} / \mathrm{s}$ ). Additive noise has often been misinterpreted as random scattering within the oil layer that results in high $H, H_{C O}, \alpha$, and low $\rho_{C O}$ and $D_{o} P$. This type of scattering has been given the name "non-Bragg". In this study, we find that there is no need to invoke such exotic scattering mechanisms - properly accounting for noise in the data means that Bragg scatter plus noise can adequately explain the observed results for both oil slicks and clean sea.

In the literature, high $H$ and $\alpha$ values have been hypothesized and reported for mineral oil slicks. However, as demonstrated in this study, the high $H$ and $\alpha$ values are most likely related to the high levels of system noise. With high SNR SAR data, e.g., from UAVSAR, the $H / \alpha$ values representing oil slick fall within the Bragg scattering region. We recommend that the $\mathrm{SNR}^{A}$ should be $\geq 10 \mathrm{~dB}$ prior to any scattering theory analysis using polarimetry. No clear threshold was identified for $\mathrm{SNR}^{A, M}$, and future analysis should therefore be performed to study this effect. Obviously, the majority of the measured signal from an oil slick should be from the oil slick itself and not other noise sources, indicating that the $\mathrm{SNR}^{A, M}$ should at least be above $0 \mathrm{~dB}$.

After noise subtraction, the features representing the oil slick had values similar to the ones from the clean sea areas, indicating that the performance of the oil slick detection might be compromised after noise subtraction. We conclude that subtracting the noise power should be performed prior to any polarimetric analysis for characterization purposes, and such measures should be used with a great deal of caution in oil slick detection.

Future studies could include a sensitivity analysis of noise contamination in various scattering models (e.g., tilted Bragg model [22]), and characterization of multiplicative noise effects on polarization features. Here we have treated MNR as another additive noise contribution, but there may still be subtle, residual correlations between MNR terms that we have not accounted for. Additionally, investigating how the noise influences the characterization of various oil types might also be valuable. Finally, we note that higher SNR instruments are needed to study oil characterizations with SAR.

\section{ACKNOWLEDGMENT}

The authors thank Yngvar Larsen at NORCE for his valuable comments and discussion in Section II, and A. Malin Johansson at UiT for valuable discussions and for helping to improve the manuscript. This research is financed in part by CIRFA (RCN Grant no. 237906). This research was carried out in part at the Jet Propulsion Laboratory, California Institute of Technology, under contract with the National Aeronautics and Space Administration (Task Order NNN13D788T). The Radarsat-2 and data Products (C)MDA LTD. 2011-2013,2015,2016. TerraSAR-X CDLR, and provided by InfoTerra. UAVSAR data can be downloaded from uavsar.jpl.nasa.gov or from the Alaska Satellite Facility (www.asf.alaska.edu).

\section{APPENDIX A}

\section{THE EXPECTED FEATURE VALUE OF NOISE}

Table IV contains the theoretical expected feature value of pure Gaussian random noise. These expected feature values could be a result of random/volume scattering from an ideal target (not the case for oil slicks), or specular reflection that has close to zero return towards the sensor and the measured signal is then dominated by system noise.

\section{A. Noise - ellipticity feature}

The ellipticity is defined as;

$$
\chi=\frac{1}{2} \sin ^{-1}\left(\frac{-S_{3}}{D o P S_{0}}\right)=\frac{1}{2} \sin ^{-1}\left(\frac{-S_{3}}{\sqrt{S_{1}^{2}+S_{2}^{2}+S_{3}^{2}}}\right) .
$$

For non-reciprocity Stokes vector, where $S_{1}=\frac{1}{2}\left(\sigma_{H H}^{n}-\right.$ $\left.\sigma_{H V}^{n}+\sigma_{V H}^{n}-\sigma_{V V}^{n}\right), S_{2}=0$, and $S_{3}=0$ the $\chi^{n}$ is:

$$
\begin{aligned}
\chi^{n} & =\frac{1}{2} \sin ^{-1}\left(\frac{-0}{\sqrt{\left(\frac{1}{2}\left(\sigma_{H H}^{n}-\sigma_{H V}^{n}+\sigma_{V H}^{n}-\sigma_{V V}^{n}\right)\right)^{2}}}\right) \\
& =\frac{1}{2} \sin ^{-1}(0)=0 .
\end{aligned}
$$

For reciprocity Stokes vector, where $S_{1}=\frac{1}{2}\left(\sigma_{H H}^{n}-\sigma_{V V}^{n}\right)$, $S_{2}=0$, and $S_{3}=-\sigma_{H V}^{n}$ the $\chi^{n}$ is:

$$
\begin{aligned}
& \chi^{n}= \frac{1}{2} \sin ^{-1}\left(\frac{-\left(-\sigma_{H V}^{n}\right)}{\sqrt{\left(-\sigma_{H V}^{n}\right)^{2}+\left(\frac{1}{2}\left(\sigma_{H H}^{n}-\sigma_{V V}^{n}\right)\right)^{2}}}\right) \\
& \stackrel{\sigma_{H H}^{n}}{\stackrel{\approx}{=} \sigma_{V V}^{n}} \frac{1}{2} \sin ^{-1}\left(\frac{1}{1}\right)=\frac{1}{2}\left(90^{\circ}\right)=45^{\circ} .
\end{aligned}
$$

In the last part of (10), we assume equal noise power in the two copolarization channels $\sigma_{H H}^{n} \approx \sigma_{V V}^{n}$ (often assumed, see e.g., [74]).

\section{APPENDIX B \\ NOISE IN THE STOKES VECTOR}

The Stokes vector from a hybrid-polarity (HP) SAR system when transmitting on right-hand $(\mathrm{R})$ circular and receiving on the linear horizontal $(\mathrm{H})$ and vertical $(\mathrm{V})$ polarization channels is given as [63]

$$
\begin{aligned}
& S_{0}=\left\langle\left|M_{R H}\right|^{2}+\left|M_{R V}\right|^{2}\right\rangle \\
& S_{1}=\left\langle\left|M_{R H}\right|^{2}-\left|M_{R V}\right|^{2}\right\rangle \\
& S_{2}=2 \Re\left\langle M_{R H} M_{R V}^{\star}\right\rangle \\
& S_{3}=-2 \Im\left\langle M_{R H} M_{R V}^{\star}\right\rangle
\end{aligned}
$$


TABLE IV

EXPECTED POLARIMETRIC FEATURE VALUE OF ADDITIVE COMPLEX GAUSSIAN WHITE NOISE. COMPLETE EXPRESSION OF THE STOKES VECTOR, BOTH FOR THE RECIPROCITY AND NON-RECIPROCITY CASES CAN BE FOUND IN APPENDIX B.

\begin{tabular}{|c|c|}
\hline Feature expression & Assumption(s) \\
\hline$P D^{n}=\sigma_{V V}^{n}-\sigma_{H H}^{n} \approx 0$ & $\sigma_{H H}^{n} \approx \sigma_{V V}^{n}$ \\
\hline$r_{C O}^{n}=\Re\left(\left\langle n_{H H} n_{V V}^{\star}\right\rangle\right) \approx 0$ & $\left\langle n_{H H} n_{V V}^{\star}\right\rangle \approx 0$ \\
\hline$\gamma_{H H / V V}^{n}=\frac{\sigma_{H H}^{n}}{\sigma_{V V}^{n}} \approx 1$ & $\sigma_{H H}^{n} \approx \sigma_{V V}^{n}$ \\
\hline$\rho_{C O}^{n}=\frac{\Re\left(\left\langle n_{H H} n_{V V}^{\star}\right\rangle\right)}{\sqrt{\sigma_{H H}^{n} \sigma_{V V}^{n}}} \approx \frac{0}{\sqrt{\sigma_{H H}^{n} \sigma_{V V}^{n}}} \approx 0$ & $\left\langle n_{H H} n_{V V}^{\star}\right\rangle \approx 0$ \\
\hline$\sigma_{\phi_{C O}^{n}}^{n}=\operatorname{std}\left(\phi_{C O}^{n}\right)=\sqrt{\operatorname{Var}\left(\phi_{C O}^{n}\right)}=\sqrt{\frac{1}{12}(2 \pi)^{2}}=\sqrt{\frac{1}{3}} \pi \approx 1.81$ & $\phi_{C O}^{n} \sim U[-\pi, \pi]$ \\
\hline $\begin{aligned} H^{n} & =-\sum_{i=1}^{3} p_{i}^{n} \log _{3} p_{i}^{n}=-\frac{1}{3} \sum_{i=1}^{3} \log _{3}(1 / 3)=-\frac{1}{3} \sum_{i=1}^{3}(-1) \approx 1 \\
p_{i}^{n} & =\frac{\lambda_{i}}{\lambda_{1}+\lambda_{2}+\lambda_{3}}=\frac{1}{3}\left(\text { eigenvalues }(\lambda) \text { of } \boldsymbol{T}_{\mathbf{3} \times \mathbf{3}}=\sigma^{n} \boldsymbol{I}_{\mathbf{3} \times \mathbf{3}}\right)\end{aligned}$ & $\begin{array}{l}\sigma_{H H}^{n} \approx \sigma_{V V}^{n} \approx \sigma_{H V}^{n} \\
\left\langle n_{j k} n_{l m}^{\star}\right\rangle=0, \text { for } j \neq l \text { or } k \neq m\end{array}$ \\
\hline $\begin{array}{l}H_{C O}^{n}=-\sum_{i=1}^{2} p_{i}^{n} \log _{2} p_{i}^{n}=-\frac{1}{2} \sum_{i=1}^{2} \log _{2}(1 / 2)=-\frac{1}{2} \sum_{i=1}^{2}(-1) \approx 1 \\
p_{i}^{n}=\frac{\lambda_{i}}{\lambda_{1}+\lambda_{2}}=\frac{1}{2}\left(\text { eigenvalues }(\lambda) \text { of } \boldsymbol{T}_{\mathbf{2} \times \mathbf{2}}=\sigma^{n} \boldsymbol{I}_{\mathbf{2} \times \mathbf{2}}\right)\end{array}$ & $\begin{array}{l}\sigma_{H H}^{n} \approx \sigma_{V V}^{n} \\
\left\langle n_{j k} n_{l m}^{\star}\right\rangle=0, \text { for } j \neq l \text { or } k \neq m\end{array}$ \\
\hline $\begin{array}{l}\alpha^{n}=\sum_{i=1}^{3} p_{i} \cos ^{-1}\left(\left|\boldsymbol{e}_{\boldsymbol{i}}(1)\right|\right)=\frac{1}{3} \sum_{i=1}^{3} \cos ^{-1}\left(\left|\boldsymbol{e}_{\boldsymbol{i}}(1)\right|\right) \\
\quad \approx \frac{1}{3}\left(\cos ^{-1}(1)+\cos ^{-1}(0)+\cos ^{-1}(0)\right) \approx \frac{1}{3}\left(90^{\circ}+90^{\circ}\right) \approx 60^{\circ} \\
\left.p_{i}^{n}=\frac{\lambda_{i}}{\lambda_{1}+\lambda_{2}+\lambda_{3}}=\frac{1}{3} \text { (eigenvalues }(\lambda) \text { of } \boldsymbol{T}_{\mathbf{3} \times \mathbf{3}}=\sigma^{n} \boldsymbol{I}_{\mathbf{3} \times \mathbf{3}}\right) \\
\boldsymbol{T}_{\mathbf{3} \times \mathbf{3}} \boldsymbol{E}=\sigma^{n} \boldsymbol{I}_{\mathbf{3} \times \mathbf{3}} \boldsymbol{E}=\boldsymbol{\Lambda} \boldsymbol{E} \Longrightarrow \boldsymbol{E}=\left[\boldsymbol{e}_{1}, \boldsymbol{e}_{2}, \boldsymbol{e}_{3}\right]=\left[\begin{array}{lll}1 & 0 & 0 \\
0 & 1 & 0 \\
0 & 0 & 1\end{array}\right]\end{array}$ & $\begin{array}{l}\sigma_{H H}^{n} \approx \sigma_{V V}^{n} \approx \sigma_{H V}^{n} \\
\left\langle n_{j k} n_{l m}^{\star}\right\rangle=0, \text { for } j \neq l \text { or } k \neq m\end{array}$ \\
\hline$D o P^{n}=\frac{\sqrt{S_{1}^{2}+S_{2}^{2}+S_{3}^{2}}}{S_{0}} \approx \begin{cases}\frac{0}{2 \sigma_{n}^{n}}=0 & \text { non-reciprocity } \\
\frac{\sigma^{n}}{2 \sigma^{n}}=0.5 & \text { reciprocity }\end{cases}$ & $\begin{array}{c}\sigma_{H H}^{n} \approx \sigma_{V V}^{n} \approx \sigma_{H V}^{n} \approx \sigma_{V H}^{n} \\
\left\langle n_{j k} n_{l m}^{\star}\right\rangle=0 \text { for } j \neq l \text { or } k \neq m\end{array}$ \\
\hline $\begin{aligned} \chi^{n}= & \frac{1}{2} \sin ^{-1}\left(\frac{-S_{3}}{D o P S_{0}}\right)=\frac{1}{2} \sin ^{-1}\left(\frac{-S_{3}}{\sqrt{S_{1}^{2}+S_{2}^{2}+S_{3}^{2}}}\right) \\
& \approx \begin{cases}\frac{1}{2} \sin ^{-1}\left(\frac{0}{\sqrt{\left(\frac{1}{2}\left(\sigma_{H H}^{n}-\sigma_{H V}^{n}+\sigma_{V H}^{n}-\sigma_{V V}^{n}\right)\right)^{2}}}\right)=0 & \text { non-reciprocity } \\
\frac{1}{2} \sin ^{-1}\left(\frac{-\left(-\sigma_{H V}^{n}\right)}{\sqrt{\left(-\sigma_{H V}^{n}\right)^{2}+\left(\frac{1}{2}\left(\sigma_{H H}^{n}-\sigma_{V V}^{n}\right)\right)^{2}}}\right)=\frac{1}{2} \sin ^{-1}(1)=45^{\circ} & \text { reciprocity }\end{cases} \end{aligned}$ & $\begin{array}{c}\text { see Appendix A-A. } \\
\left\langle n_{j k} n_{l m}^{\star}\right\rangle=0 \text { for } j \neq l \text { or } k \neq m\end{array}$ \\
\hline
\end{tabular}

where the $M_{R H}$ and $M_{R V}$ are the measured HP complex scattering coefficients and can be expressed as [77]

$$
\begin{aligned}
& M_{R H}=\frac{1}{\sqrt{2}}\left(M_{H H}-j M_{V H}\right) \\
& M_{R V}=\frac{1}{\sqrt{2}}\left(M_{H V}-j M_{V V}\right)
\end{aligned}
$$

using the linear complex scattering coefficients. The Stokes vector can be expressed using the linear complex scattering coefficients in the following way (without assuming reciprocity);

$$
\begin{aligned}
S_{0} & =\frac{1}{2}\left\langle\left(\left|M_{H H}\right|^{2}+\left|M_{V V}\right|^{2}+\left|M_{H V}\right|^{2}+\left|M_{V H}\right|^{2}\right)\right. \\
& \left.-2 \Im\left(M_{H H} S_{V H}^{\star}\right)-2 \Im\left(M_{H V} M_{V V}^{\star}\right)\right\rangle \\
S_{1} & =\frac{1}{2}\left\langle\left(\left|M_{H H}\right|^{2}-\left|M_{V V}\right|^{2}-\left|M_{H V}\right|^{2}+\left|M_{V H}\right|^{2}\right)\right. \\
& \left.-2 \Im\left(M_{H H} M_{V H}^{\star}\right)+2 \Im\left(M_{H V} M_{V V}^{\star}\right)\right\rangle \\
S_{2} & =\left\langle\Re\left(M_{H H} M_{H V}^{\star}\right)+\Re\left(M_{V H} M_{V V}^{\star}\right)-\Im\left(M_{H H} M_{V V}^{\star}\right)\right. \\
& \left.+\Im\left(M_{V H} M_{H V}^{\star}\right)\right\rangle \\
S_{3} & =\left\langle\Im\left(M_{H H} M_{H V}^{\star}\right)+\Im\left(M_{V H} M_{V V}^{\star}\right)+\Re\left(M_{H H} M_{V V}^{\star}\right)\right. \\
& \left.-\Re\left(M_{V H} M_{H V}^{\star}\right)\right\rangle .
\end{aligned}
$$

The Stokes vector with the noise power, i.e., writing out the $M_{p q}=S_{p q}+\sigma_{p q}^{n}$, where $p$ and $q$ is polarization on transmits and receive is

$$
\begin{aligned}
S_{0} & =\frac{1}{2}\left\langle\left(\left|S_{H H}\right|^{2}+\left|S_{V V}\right|^{2}+\left|S_{H V}\right|^{2}+\left|S_{V H}\right|^{2}+\sigma_{H H}^{n}\right.\right. \\
& \left.\left.+\sigma_{H V}^{n}+\sigma_{V H}^{n}+\sigma_{V V}^{n}\right)-2 \Im\left(S_{H H} S_{V H}^{\star}\right)-2 \Im\left(S_{H V} S_{V V}^{\star}\right)\right\rangle \\
S_{1} & =\frac{1}{2}\left\langle\left(\left|S_{H H}\right|^{2}-\left|S_{V V}\right|^{2}-\left|S_{H V}\right|^{2}+\left|S_{V H}\right|^{2}+\sigma_{H H}^{n}\right.\right. \\
& \left.\left.-\sigma_{H V}^{n}+\sigma_{V H}^{n}-\sigma_{V V}^{n}\right)-2 \Im\left(S_{H H} S_{V H}^{\star}\right)+2 \Im\left(S_{H V} S_{V V}^{\star}\right)\right\rangle \\
S_{2} & =\left\langle\Re\left(S_{H H} S_{H V}^{\star}\right)+\Re\left(S_{V H} S_{V V}^{\star}\right)-\Im\left(S_{H H} S_{V V}^{\star}\right)\right. \\
& \left.+\Im\left(S_{V H} S_{H V}^{\star}\right)\right\rangle \\
S_{3} & =\left\langle\Im\left(S_{H H} S_{H V}^{\star}\right)+\Im\left(S_{V H} S_{V V}^{\star}\right)+\Re\left(S_{H H} S_{V V}^{\star}\right)\right. \\
& \left.-\Re\left(S_{V H} S_{H V}^{\star}\right)\right\rangle .
\end{aligned}
$$

In [63], the author expressed the Stokes vector using elements from the $3 \times 3$ covariance matrix assuming reciprocity $\left(S_{H V}=S_{V H}\right)$. The Stokes vector with the noise elements 
and assuming reciprocity becomes

$$
\begin{aligned}
S_{0} & =\frac{1}{2}\left\langle\left(\left|S_{H H}\right|^{2}+\left|S_{V V}\right|^{2}+2\left|S_{H V}\right|^{2}+\sigma_{H H}^{n}+2 \sigma_{H V}^{n}\right.\right. \\
& \left.\left.+\sigma_{V V}^{n}\right)-2 \Im\left(S_{H H} S_{H V}^{\star}\right)-2 \Im\left(S_{H V} S_{V V}^{\star}\right)\right\rangle \\
S_{1} & =\frac{1}{2}\left\langle\left(\left|S_{H H}\right|^{2}-\left|S_{V V}\right|^{2}\right)-2 \Im\left(S_{H H} S_{H V}^{\star}\right)+\sigma_{H H}^{n}\right. \\
& \left.-\sigma_{H V}^{n}+\sigma_{H V}^{n}-\sigma_{V V}^{n}+2 \Im\left(S_{H V} S_{V V}^{\star}\right)\right\rangle \\
S_{2} & =\left\langle\Re\left(S_{H H} S_{H V}^{\star}\right)+\Re\left(S_{H V} S_{V V}^{\star}\right)-\Im\left(S_{H H} S_{V V}^{\star}\right)\right\rangle \\
S_{3} & =\left\langle\Im\left(S_{H H} S_{H V}^{\star}\right)+\Im\left(S_{H V} S_{V V}^{\star}\right)+\Re\left(S_{H H} S_{V V}^{\star}\right)\right. \\
& \left.-\left|S_{H V}\right|^{2}-\sigma_{H V}^{n}\right\rangle .
\end{aligned}
$$

As seen in the equation above, an extra noise component in $S_{3}$ is present, which was not present when reciprocity was not assumed.

\section{APPENDIX C}

\section{ADDING NOISE TO THE UAVSAR}

We want to compare measured additive noise with simulated noise in the SAR measurements. This is performed by generating independently, for each polarization channel, complex random Gaussian white noise ( $N$ term in equation (3)) with zero mean and variance equal to the noise power added. Addition of simulated noise to the measured signal is performed on the complex scattering coefficient prior to any filtering and feature calculation, i.e.,

$$
\hat{S}_{p q}=M_{p q}+\frac{1}{\sqrt{2}}(X+j Y), X \sim N\left(0, \sigma_{n}\right), Y \sim N\left(0, \sigma_{n}\right)
$$

where $\sigma_{n}$ is equal to the NESZ $+\Delta_{n}$. The $\frac{1}{\sqrt{2}}$ accounts for the two components in the complex signal. $\Delta_{n}$ increases with $+1 \mathrm{~dB}$ for every iteration until the $\mathrm{SNR}$ is equal to $-10 \mathrm{~dB}$. For example, if NESZ is $-45 \mathrm{~dB}$, the first iteration has a noise variance (in $\mathrm{dB}$ ) equal to $-44 \mathrm{~dB}$. Note that this procedure is only applied to the UAVSAR, due to its high initial SNR for both the oil slick and the clean sea regions.

\section{APPENDIX D}

\section{THE VALIDITY OF THE NOMINAL NOISE FLOOR}

To verify the NESZ provided in the product files, we estimate the NESZ from both RS-2 and UAVSAR data using two methods [75], [76], based on the theory in [73], [74]. Once the estimates of the NESZ are obtained, a comparison with the nominal NESZ values from the product file is carried out. The reason for estimating the NESZ is to verify the accuracy of the nominal NESZ, since the NESZ is used to estimate the SNR.

The authors of [75] suggested an approach to estimate the NESZ using a method based on the minimum eigenvalue of the $4 \times 4$ sample coherency matrix, named the eigenvaluebased (EB) estimator of NESZ, i.e., $\mathrm{NESZ}_{E B}$. The fourth (minimum) eigenvalue represents the noise power, i.e., the NESZ. The second method tested was suggested in [76], and uses the maximum likelihood (ML) to estimate the NESZ, i.e., $\mathrm{NESZ}_{M L}$. To estimate the NESZ based on the ML estimator, a given number of samples are needed (see [76] for more details). The number of samples is given within a window that steps across the two cross-polarization coefficients (HV and $\mathrm{VH}$ ). The same is the case for the EB estimator, since the sample coherency matrix is calculated using a smoothing filter with a given window size, i.e., number of samples. To evaluate the robustness in terms of the window size, the EM and the ML estimator of the NESZ is carried out with several window sizes using an averaging filter mask.

The estimation of the NESZ is performed over a clean sea area across the full range within each RS-2 and UAVSAR scene. Figs. 10 and 11 show the two estimators using only one RS-2 scene acquired 8 June 2011 and one UAVSAR scene acquired 11 June 2015. The estimated NESZ based on the EB-estimator (the left panel of Fig. 10) seems to converge towards a given value as the window size increases. The EB estimator is very sensitive to the window size, as expected based on the study in [76], where the authors show that the EB estimator is biased. Therefore, [76] suggested an unbiased and more efficient estimator of the noise power, namely the ML estimator. The estimator's sample size independence is observed in the right panel of Figs. 10 and 11.

The estimated NESZ of both the EB (with large window size) and the ML estimator is about $\pm 1 \mathrm{~dB}$ from the nominal NESZ values for all the RS-2 scenes used in this study. Therefore, we consider the nominal NESZ values from RS2 trustworthy to use in the analysis. Unfortunately, since no quad-polarimetric data are available in the TS-X products, the ML and EB methods cannot be applied. The estimated NESZ of the UAVSAR is higher than the nominal values by more than $10 \mathrm{~dB}$ for some incidence angles. This is consistent with the influence of ISLR as a multiplicative noise term (see Table I), reducing the effective SNR for UAVSAR data by up to $15 \mathrm{~dB}$ as seen in Fig. 3. The cross-talk contamination/leakage from the copolarization into the cross-polarization channels may also contribute to this high deviation between the nominal and the estimated NESZ. The influence of the multiplicative noise terms is not observed in the estimated NESZ for RS-2, as the estimated NESZ values are similar to the nominal ones. Further investigation of the absence of the multiplicative noise terms is therefore encouraged.
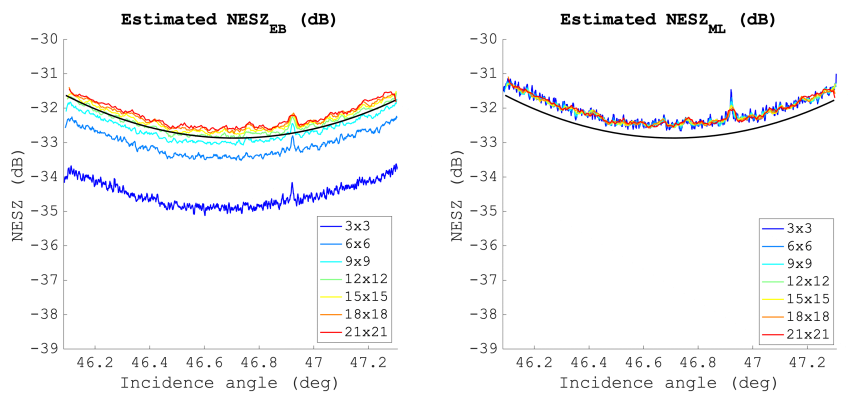

Fig. 10. Estimation of the noise powers $\mathrm{NESZ}_{E B}$ and $\mathrm{NESZ}_{M L}$ of one RS-2 scene (8 June 2011 at 05:59 UTC) using various window sizes when smoothing with an averaging filter mask. The black continuous line shows the nominal NESZ values for the given RS-2 product.

\section{REFERENCES}

[1] A.-B. Salberg, Ø. Rudjord, and A. H. S. Solberg, "Oil Spill Detection in Hybrid-Polarimetric SAR Images," IEEE Trans. Geosci. Remote Sens., vol. 52, no. 10, pp. 6521 - 6533, Oct. 2014. 

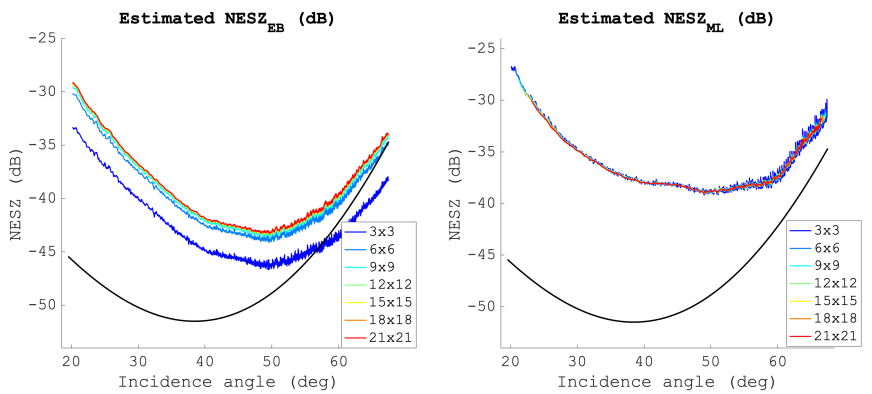

Fig. 11. Estimation of the noise powers $\mathrm{NESZ}_{E B}$ and $\mathrm{NESZ}_{M L}$ of one UAVSAR scene (11 June 2015 at 08:46 UTC) using various window sizes when smoothing with an averaging filter mask. The black continuous line shows the nominal NESZ values for the given UAVSAR product.

[2] S. Skrunes, C. Brekke, and T. Eltoft, "Characterization of marine surface slicks by Radarsat-2 multipolarization features," IEEE Trans. Geosci. Remote Sens., vol. 52, no. 9, pp. 5302-5319, Sep. 2014.

[3] B. Minchew, "Determining the mixing of oil and sea water using polarimetric Synthetic Aperture Radar," Geophys. Res. Lett., vol. 39, no. 16, Aug. 2012.

[4] B. Fiscella, A. Giancaspro, F. Nirchio, P. Pavese, and P. Trivero, "Oil spill detection using marine SAR images," Int. J. Remote Sens., vol. 21, no. 18 , pp. $3561-3566,2000$

[5] R. Shirvany, M. Chabert, and J.-Y. Tourneret, "Ship and Oil-Spill Detection using the Degree of Polarization in Linear and Hybrid/Compact Dual-Pol SAR," IEEE J. Sel. Topics Appl. Earth Observ. Remote Sens., vol. 5, no. 3, pp. 885 - 892, Jun. 2012.

[6] M. Migliaccio, A. Gambardella, and M. Tranfaglia, "SAR polarimetry to observe oil spills," IEEE Trans. Geosci. Remote Sens., vol. 45, no. 2 , pp. 506 - 511, Feb. 2007

[7] S. Angelliaume, O. Boisot, and C.-A. Guérin, "Dual-Polarized L-band SAR Imagery for Temporal Monitoring of Marine Oil Slick Concentration," Remote Sens., vol. 10, no. 1012, pp. 1-17, Jun. 2018.

[8] C. Oliver and S. Quegan, Understanding Synthetic Aperture Radar Images. SciTech Publishing Inc., 2004.

[9] A. Freeman, "The effects of noise on polarimetric SAR data," IEEE Geoscience and Remote Sensing Symposium, 1993. IGARSS '93. Better Understanding of Earth Environment., International, vol. 2, pp. 799802, 1993

[10] B. Minchew, C. E. Jones, and B. Holt, "Polarimetric Analysis of Backscatter From the Deepwater Horizon Oil Spill Using L-Band Synthetic Aperture Radar," IEEE Trans. Geosci. Remote Sens., vol. 50, no. 10, pp. 3812-3830, Oct. 2012.

[11] D. Latini, F. D. Frate, and C. E. Jones, "Multi-frequency and polarimetric quantitative analysis of the Gulf of Mexico oil spill event comparing different SAR systems," Remote Sens. Environ., vol. 183, pp. 26 - 42, 2016.

[12] S. Skrunes, C. Brekke, C. E. Jones, M. M. Espeseth, and B. Holt, "Effect of wind direction and incidence angle on polarimetric SAR observations of slicked and unslicked sea surfaces," Remote Sens. Environ., vol. 213, pp. 73 - 91, Aug. 2018.

[13] S. Angelliaume, P. C. Dubois-Fernandez, C. E. Jones, B. Holt, B. Minchew, E. Amri, and V. Miegebielle, "SAR imagery for detecting sea surface slicks: Performance assessment of polarization-dependent parameters," IEEE Trans. Geosci. Remote Sens., vol. 56, no. 8, pp. 4237 - 4257, Aug. 2018.

[14] S. Skrunes, C. Brekke, C. E. Jones, and B. Holt, "A Multisensor Comparison of Experimental Oil Spills in Polarimetric SAR for High Wind Conditions," IEEE J. Sel. Topics Appl. Earth Observ. Remote Sens., vol. 9, no. 11, pp. 4948-4961, Nov. 2016.

[15] M. M. Espeseth, S. Skrunes, C. E. Jones, C. Brekke, B. Holt, and A. P. Doulgeris, "Analysis of Evolving Oil Spills in Full-Polarimetric and Hybrid-Polarity SAR," IEEE Trans. Geosci. Remote Sens., vol. 55, no. 7, pp. 4190-4210, July 2017.

[16] A. Buono, C. R. De Macedo, F. Nunziata, D. Velotto, and M. Migliaccio, "Analysis on the effects of sar imaging parameters and environmental conditions on the standard deviation of the co-polarized phase difference measured over sea surface," Remote Sens., vol. 11, no. 1, 2018.

[17] I. G. Cumming and F. H. Wong, Digital Processing of Synthetic Aperture Radar data. Artech House, 2005.
[18] T. Fritz and M. Eineder, "TerraSAR-X Ground Segment Basic Product Specification Document (doc: TX-GS-DD-3302)," DLR, Oct. 2013.

[19] A. Luscombe, "Radarsat-2 image quality and mode maintenance and enhancement," EOS SAR Cal/Val Working Group Meeting, Pasadena, 2009.

[20] T. L. Ainsworth, L. Ferro-Famil, and J.-S. Lee, "Orientation angle preserving a posteriori polarimetric SAR calibration," IEEE Trans. Geosci. Remote Sens., vol. 44, no. 4, pp. 994-1003, Apr. 2006.

[21] S. Quegan, "A unified algorithm for phase and cross-talk calibration of polarimetric data-theory and observations," IEEE Trans. Geosci. Remote Sens., vol. 32, no. 1, pp. 89-99, Jan. 1994.

[22] G. R. Valenzuela, "Theories for the interaction of electromagnetic and oceanic waves - a review," Boundary-Layer Meteorology, vol. 12, no. 1-4, pp. 61-85, Jan. 1978.

[23] R. Touzi, R. K. Hawkins, and S. Cote, "High-precision assessment and calibration of polarimetric RADARSAT-2 SAR using transponder measurements," IEEE Trans. Geosci. Remote Sens., vol. 51, no. 1, pp. 487-503, Jan. 2013

[24] A. P. Luscombe, K. Chotoo, and B. D. Huxtable, "Polarimetric calibration for RADARSAT-2," IGARSS 2000. IEEE 2000 Int. Geosci. Remote Sens. Symp. (IGARSS) Taking the Pulse of the Planet: The Role of Remote Sensing in Managing the Environment. Proceedings (Cat. No.00CH37120), vol. 5, pp. 2197-2199, 2000.

[25] D. Williams, Y. Wang, M. Chabot, P. L. Dantec, R. Caves, Y. Wu, K. James, A. Thompson, and C. Vigneron. (2015) Radarsat-2 Image Quality and Calibration Update 2015. [Online]. Available: http://SARcv.ceos.org/site_media/media/documents/03_RADARSAT2_Image_Quality_and_Calibration_Update_CEOS_2015.pdf

[26] A. G. Fore, B. D. Chapman, B. P. Hawkins, S. Hensley, C. E. Jones, T. R. Michel, and R. J. Muellerschoen, "UAVSAR Polarimetric Calibration," IEEE Trans. Geosci. Remote Sens., vol. 53, no. 6, pp. 3481-3491, Jun. 2015.

[27] R. Touzi, P. W. Vachon, and J. Wolfe, "Requirement on antenna crosspolarization isolation for the operational use of c-band sar constellations in maritime surveillance," IEEE Geosci. Remote Sens. Lett., vol. 7, no. 4, pp. 861-865, Oct 2010.

[28] A. Freeman, J. J. van Zyl, J. D. Klein, H. A. Zebker, and Y. Shen, "Calibration of stokes and scattering matrix format polarimetric sar data," IEEE Trans. Geosci. Remote Sens., vol. 30, no. 3, pp. 531-539, May 1992.

[29] D. Williams, Y. Wang, G. Fitzgerald, R. Caves, M. Chabot, N. Gibb, Y. Wu, A. Thompson, P. Allan, and C. Vigneron. (2017) Radarsat-2 Image Quality and Calibration Update 2017. [Online]. Available: http://SARcv.ceos.org/site_media/media/documents/RADARSAT2_Image_Quality_and_Calibration_Update_CEOS_2017.pdf

[30] S. Hensley, S. Oveisgharan, S. Saatchi, M. Simard, R. Ahmed, and Z. Haddad, "An error model for biomass estimates derived from polarimetric radar backscatter," IEEE Trans. Geosci. Remote Sens., vol. 52, no. 7, pp. 4065-4082, Jul. 2014

[31] "Radarsat-2 product description," MacDonald, Dettwiler and Associates Ltd., Rickmond, BC, USA, RN-SP-52-1238, vol. Issue 1/13, 2016.

[32] M. Vespe and H. Greidanus, "SAR image quality assessment and indicators for vessel and oil spill detection," IEEE Trans. Geosci. Remote Sens., vol. 50, no. 11, pp. 4726-4734, Nov 2012.

[33] S. Hensley. (2015) 3rd Advanced Course on Radar Polarimetry, ESA-ESRIN. [Online]. Available: http://seom.esa.int/polarimetrycourse2015/files/UAVSAR_SHensley.pdf

[34] J. Mittermayer, M. Younis, R. Metzig, S. Wollstadt, J. M. Martinez, and A. Meta, "TerraSAR-X system performance characterization and verification," IEEE Trans. Geosci. Remote Sens., vol. 48, no. 2, pp. 660-676, Feb. 2010.

[35] S. Hensley, "New Missions, UAVSAR," in Proc. POLinSAR, Frascati, Italy, 2015

[36] X. Li, H. Qi, B. Hua, H. Lei, and W. Yu, "A study of spaceborne SAR raw data compression error based on a statistical model of quantization interval transfer probability," Science China Information Sciences, vol. 53, no. 11, pp. 2352-2362, Nov 2010.

[37] S. R. Cloude, Polarisation Applications in Remote Sensing, First, Ed. Oxford University Press, 2010.

[38] J. Wright, "Backscattering from capillary waves with application to sea clutter," IEEE Trans. Antennas Propag., vol. 14, no. 6, pp. 749-754, Nov. 1966.

[39] I. Hajnsek, E. Pottier, and S. R. Cloude, "Inversion of surface parameters from polarimetric SAR," IEEE Trans. Geosci. Remote Sens., vol. 41, no. 4, pp. 727-744, Apr. 2003. 
[40] A. Iodice, A. Natale, and D. Riccio, "Retrieval of soil surface parameters via a polarimetric two-scale model," IEEE Trans. Geosci. Remote Sens., vol. 49, no. 7, pp. 2531-2547, Jul. 2011.

[41] A. Buono, F. Nunziata, and M. Migliaccio, "New generation polarimetric SARs for sea oil slick observation: Full-polarimetric vs compactpolarimetric architectures," in 2016 Proc. IEEE Int. Geosci. Remote Sens. Symp. (IGARSS), July 2016, pp. 2223-2226.

[42] J. Yin, J. Yang, Z. Zhou, and J. Song, "The Extended Bragg Scattering Model-Based Method for Ship and Oil-Spill Observation Using Compact Polarimetric SAR," IEEE J. Sel. Topics Appl. Earth Observ. Remote Sens., vol. 8, no. 8, pp. 3760-3772, Aug 2015.

[43] A. Buono, F. Nunziata, C. R. de Macedo, D. Velotto, and M. Migliaccio, "A Sensitivity Analysis of the Standard Deviation of the Copolarized Phase Difference for Sea Oil Slick Observation," IEEE Trans. Geosci. Remote Sens., vol. 57, no. 4, pp. 2022-2030, April 2019.

[44] A. Buono, F. Nunziata, and M. Migliaccio, "Analysis of Full and Compact Polarimetric SAR Features Over the Sea Surface," IEEE Geosci. Remote Sens. Lett., vol. 13, no. 10, pp. 1527-1531, Oct 2016.

[45] W. Alpers, B. Holt, and K. Zeng, "Oil spill detection by imaging radars: Challenges and pitfalls," Remote Sens. Environ., vol. 201, pp. 133-147, Nov. 2017.

[46] J.-S. Lee and E. Pottier, Polarimetric Radar Imaging, B. J. Thompson, Ed. CRC Press Taylor and Francis Group, 2009, no. 42-45.

[47] S. Skrunes, C. Brekke, T. Eltoft, and V. Kudryavtsev, "Comparing NearCoincident C- and X-Band SAR Acquisitions of Marine Oil Spills," IEEE Trans. Geosci. Remote Sens., vol. 53, no. 4, pp. 1958-1975, Apr. 2015.

[48] V. Kudryavtsev, B. Chapron, A. Myasoedova, F. Collard, and J. Johannessen, "On dual co-polarized SAR measurements of the ocean surface," IEEE Geosci. Remote Sens. Lett., vol. 10, no. 4, pp. 761-765, Jul. 2013.

[49] O. Boisot, S. Angelliaume, and C. Guérin, "Marine Oil Slicks Quantification From L-band Dual-Polarization SAR Imagery," IEEE Trans. Geosci. Remote Sens., vol. 57, no. 4, pp. 2187-2197, April 2019.

[50] H. Li, W. Perrie, and J. Wu, "Retrieval of Oil-Water Mixture Ratio at Ocean Surface Using Compact Polarimetry Synthetic Aperture Radar," Remote Sens., vol. 11, no. 7, 2019.

[51] H. Lin, Q. Xu, and Q. Zheng, "An overview on SAR measurements of sea surface wind," Progress in Natural Science, vol. 18, no. 8, pp. 913 - 919, Aug. 2008

[52] D. Kasilingam, D. Schuler, J.-S. Lee, and S. Malhotra, "Modulation of polarimetric coherence by ocean features," IEEE Int. Geosci. Remote Sens. Symp., vol. 1, pp. 432-434, Jun. 2002.

[53] M. R. Drinkwater, R. Kwok, E. Rignot, H. Israelsson, R. G. Onstott, and D. P. Winebrenner, "Chapter 24. Potential Applications of Polarimetry to the Classification of Sea Ice," Microwave Remote Sensing of Sea Ice, Geophysical Monograph 68, American Geophysical Union, Jan. 1992.

[54] D. Velotto, M. Migliaccio, F. Nunziata, and S. Lehner, "Dual-Polarized TerraSAR-X Data for Oil-Spill Observation," IEEE Trans. Geosci. Remote Sens., vol. 49, no. 12, pp. 4751-4762, Dec. 2011.

[55] M. Migliaccio, F. Nunziata, and A. Gambardella, "On the co-polarized phase difference for oil spill observation," Int. J. Remote Sens., vol. 30, no. 6, pp. 1587-1602, Apr. 2009.

[56] S. Singha, R. Ressel, D. Velotto, and S. Lehner, "A combination of traditional and polarimetric features for oil spill detection using TerraSAR-X," IEEE J. Sel. Topics Appl. Earth Observ. Remote Sens., vol. 9, no. 11, pp. 4979-4990, Nov. 2016.

[57] M. Migliaccio, F. Nunziata, A. Montuori, X. Li, and W. G. Pichel, "A multifrequency polarimetric SAR processing chain to observe oil fields in the Gulf of Mexico," IEEE Trans. Geosci. Remote Sens., vol. 49, no. 12, pp. 4729-4737, Dec. 2011.

[58] F. Nunziata, C. R. de Macedo, A. Buono, D. Velotto, and M. Migliaccio, "On the analysis of a time series of X-band TerraSAR-X SAR imagery over oil seepages," Int. J. Remote Sens., vol. 40, no. 9, pp. 3623-3646, 2019.

[59] S. R. Cloude and E. Pottier, "An entropy based classification scheme for land applications of polarimetric SAR," IEEE Trans. Geosci. Remote Sens., vol. 35, no. 1, pp. 68-78, Jan. 1997.

[60] W. Tian, Y. Shao, J. Yuan, S. Wang, and Y. Liu, "An experiment for oil spill recognition using Radarsat-2 image," IEEE Int. Geosci. Remote Sens. Symp., pp. 2761-2764, Jul. 2010.

[61] B. Zhang, W. Perrie, X. Li, and W. G. Pichel, "Mapping sea surface oil slicks using Radarsat-2 quad-polarization SAR image," Geophys. Res. Lett., vol. 38, Jul. 2011

[62] M. Migliaccio, A. Gambardella, F. Nunziata, M. Shimada, and O. Isoguchi, "The PALSAR Polarimetric Mode for Sea Oil Slick Observation," IEEE Trans. Geosci. Remote Sens., vol. 47, no. 12, pp. 4032-4041, Dec. 2009
[63] R. K. Raney, "Hybrid-Polarity SAR Architecture," IEEE Trans. Geosci. Remote Sens., vol. 45, no. 11, pp. 3397-3404, Nov. 2007.

[64] K. Raney, J. T. S. Cahill, G. W. Patterson, and D. B. J. Bussey, "The m-chi Decomposition of Hybrid Dual-Polarimetric Radar Data with Application to Lunar Craters," J. Geophys. Res., vol. 117, no. E12, May 2012.

[65] A. Buono, F. Nunziata, M. Migliaccio, and X. Li, "Polarimetric analysis of compact-polarimetry SAR architectures for sea oil slick observation," IEEE Trans. Geosci. Remote Sens., vol. 54, no. 10, pp. 5862-5874, Oct. 2016.

[66] Y. Zhang, Y. Li, X. S. Liang, and J. Tsou, "Comparison of oil spill classifications using fully and compact polarimetric SAR images," Applied Sciences, vol. 7, no. 2, p. 193, Feb. 2017.

[67] F. Nunziata, A. Gambardella, and M. Migliaccio, "On the degree of polarization for SAR sea oil slick observation," ISPRS Journal of Photogrammetry and Remote Sensing, vol. 78, pp. 41 - 49, 2013. [Online]. Available: http://www.sciencedirect.com/science/article/pii/S0924271613000221

[68] S. Cloude, D. Goodenough, and H. Chen, "Compact Decomposition Theory," IEEE Trans. Geosci. Remote Sens., vol. 9, no. 1, pp. 28-32, Jan. 2012.

[69] H. Li, W. Perrie, Y. He, J. Wu, and X. Luo, "Analysis of the Polarimetric SAR Scattering Properties of Oil-Covered Waters," IEEE J. Sel. Topics Appl. Earth Observ. Remote Sens., vol. 8, no. 8, pp. 3751-3759, Aug. 2015.

[70] S. Skrunes, C. Brekke, and M. M. Espeseth, "Assessment of the RISAT-1 FRS-2 mode for oil spill observation," in 2017 IEEE Int. Geosci. Remote Sens. Symp. (IGARSS), July 2017, pp. 1024-1027.

[71] B. Holt, "SAR imaging of the ocean surface," Synthetic Aperture Radar Marine User's Manual, no. 25-80, C. Jackson and J. Apel, Eds. U.S. Department of Commerce, National Oceanic and Atmospheric Administration, C. Jackson and J. Apel, 2004.

[72] F. Nunziata, A. Gambardella, and M. Migliaccio, "On the Mueller scattering matrix for SAR sea oil slick observation," IEEE Geosci. Remote Sens. Lett., vol. 5, no. 4, pp. 691-695, Oct. 2008.

[73] A. Freeman, J. Villasenor, and J. D. Klein, "Multi-frequency and polarimetric radar backscatter signatures for discrimination between agricultural crops at the Flevoland experimental test site." AIRSAR Workshop, no. 92-28018, 1991.

[74] A. Freeman, "An Algorithm for estimating the noise levels in polarimetric SAR image data," Int. J. Remote Sens., vol. 15, no. 9, pp. 1791-1798, 1993.

[75] S. Hajnsek, K. P. Papathanassiou, and S. R. Cloude, "Removal of additive noise in polarimetric eigenvalue processing," IGARSS 2001. Scanning the Present and Resolving the Future. Proceedings. IEEE Int. Geosci. Remote Sens. Symp., vol. 6, pp. 2778-2780, 2001.

[76] M. Villano, "SNR and noise variance estimation in polarimetric SAR data," IEEE Geosci. Remote Sens. Lett., vol. 11, no. 1, pp. 278-282, 2014.

[77] P. Dubois-Fernandez, J.-C. Souyris, S. Angelliaume, and F. Garestier, "The Compact Polarimetry Alternative for Spaceborne SAR at Low Frequency," IEEE Trans. Geosci. Remote Sens., vol. 46, no. 10, Oct. 2008. 